\title{
Enabling LTE and WiFi Coexisting in 5 GHz for Efficient Spectrum Utilization
}

\author{
Hongyu Sun, ${ }^{1,2}$ Zhiyi Fang, ${ }^{1}$ Qun Liu, ${ }^{3}$ Zheng Lu, ${ }^{1,2}$ and Ting $\mathrm{Zhu}^{2}$ \\ ${ }^{1}$ College of Computer Science and Technology, Jilin University, Changchun, China \\ ${ }^{2}$ Computer Science and Electrical Engineering, University of Maryland, Baltimore, MD, USA \\ ${ }^{3}$ Network Center, Jilin University, Changchun, China
}

Correspondence should be addressed to Qun Liu; liuqun@jlu.edu.cn and Ting Zhu; zt@umbc.edu

Received 28 March 2016; Revised 1 October 2016; Accepted 22 November 2016; Published 9 February 2017

Academic Editor: Kyandoghere Kyamakya

Copyright (C) 2017 Hongyu Sun et al. This is an open access article distributed under the Creative Commons Attribution License, which permits unrestricted use, distribution, and reproduction in any medium, provided the original work is properly cited.

\begin{abstract}
Due to the increasing mobile traffic demands in cellular network, researchers have proposed the coexistence of LTE and WiFi technologies in $5 \mathrm{GHz}$ unlicensed bands. Therefore, how to efficiently utilize the spectrum in $5 \mathrm{GHz}$ becomes extremely important. To avoid the channel access conflicts, current LTE Unlicensed (LTE-U) technology introduces the duty cycle of LTE, while LicenseAssisted Access (LAA) technology introduces Listen-Before-Talk (LBT) mechanism. While these two technologies improve the spectrum utilization by using time division access schema, we believe that more efficient spectrum utilization can be achieved by enabling simultaneous transmissions from LTE and WiFi. In this paper, we propose a novel method (i.e., Low Amplitude Stream Injection (LASI) method) to enable the simultaneous transmissions of WiFi and LTE frames in the same channel and recover the data from the conflicts. To further utilize the LASI method, we introduce the Conflict-Tolerant Channel Allocation (CTCA) algorithm to optimize the channel allocation and achieve more efficient spectrum utilization in $5 \mathrm{GHz}$. Extensive simulation results show that our approach achieves lower latency and higher throughput. Compared with the state-of-the-art LTE-U and LAA technologies, our approach can improve the spectrum efficiency 2.9 times.
\end{abstract}

\section{Introduction}

As the number of mobile devices (e.g., smartphones and tablets) increases, mobile traffic demand is increasing significantly in recent years. According to the global traffic forecast by Cisco Visual Networking Index (VNI) [1], global mobile data traffic reached 3.7 exabytes per month by the end of 2015, up from 2.1 exabytes per month by the end of 2014. Furthermore, the mobile data traffic will continue to grow and reach 30.6 exabytes per month by 2020 at a compound annual growth rate (GAGR) of $53 \%$. However, the spectrum utilized to offload these mobile traffic is scarce resource according to the regulations of ITU (International Telecommunication Union) [2]. Therefore, it is urgent for the research communities to look for new solutions to solve the increasing mobile traffic.

One of the most promising solutions for increasing capacities is utilizing ISM spectrum in $5 \mathrm{GHz}$. Currently, IEEE developed 802.11ac [3] and also extent $802.11 \mathrm{n}$ to $5 \mathrm{GHz}$ to offload the increasing mobile traffic, while there has been a recent push by many major companies, such as Qualcomm, Huawei, Nokia, Ericsson, T-Mobile, and NTT, to deploy LTE devices in $5 \mathrm{GHz}$ [4-7] as the addition of the existing licensed spectrum. The first standard has been published by 3GPP in 2014 [8].

How to efficiently assign channels between LTE and WiFi devices comes to a key challenge to achieve the coexistence and collaborations between multiple WiFi access points and LTE eNodeBs. The existing channel management works such as [9-13] only focus on how to allocate channels in the homologous WiFi networks.

The most related works used to manage channels between LTE and WiFi are LTE Unlicensed (LTE-U) proposed by Qualcomm in [14] and License-Assisted Access (LAA) proposed by $3 \mathrm{GPP}$ in release 13 [8]. These two methods could achieve the coexistence of LTE and WiFi devices when they share the same spectrum. However, both LTE-U and LAA are time division multiple access methods by which LTE and 
WiFi must use the spectrum alternatively in time domain. However, the time division only allows LTE and WiFi devices use the channel alternatively, which could not optimize the spectrum utilization and also introduce time delay. Therefore, we propose Low Amplitude Stream Injection (LASI) and Conflict-Tolerant Channel Allocation (CTCA) to enable the coexistence of LTE and WiFi with high performance and better channel utilization. Specifically, our contributions are as follows.

(i) Compared with state-of-the-art LTE-U/LAA or LTEU/LAA based works, we propose enabling LTE and WiFi devices to allocate the channel simultaneously in time domain and apply Low Amplitude Stream Injection (LASI) method for resolving interference between them when they transit together. We also proved the correctness of LASI method in theoretical level. Theoretically, LASI could increase the channel utilization efficiency especially in dense deployed scenarios since it has great probability that LTE and WiFi devices allocate the same channel in these scenarios.

(ii) In order to further improve the channel utilization between LTE and WiFi devices in both low and high-density deployed scenarios, we further propose Conflict-Tolerant Channel Allocation (CTCA) to adaptively choose channel for optimizing both interference and network performances. CTCA defines a parameter to help APs decide which channel to allocate for transmission according to current topologies. Theoretically, CTCA could adaptively optimize interference and performance in both low and highdensity deployed scenarios.

(iii) Extensive simulation results show that our approach achieves lower latency and higher throughput. Compared with state-of-the-art LTE-U and LAA technologies, our approach can improve the spectrum efficiency 2.9 times.

The rest of this paper is organized as follows: Section 2 discusses the related work. Section 3 introduces the background of LTE and WiFi coexistence researches. Section 4 presents the motivation behind the work. Section 5 defines the network model and assumptions. Section 6 introduces our main design, followed by the evaluation in Section 7. Section 8 concludes the paper.

\section{Related Work}

We classify related work into (i) spectrum sharing between homogeneous technologies, (ii) spectrum sharing between heterogeneous technologies, and (iii) the current spectrum sharing methods between LTE and WiFi in unlicensed $5 \mathrm{GHz}$ bands.

(i) Spectrum Sharing between Homogeneous Technologies. The current spectrum sharing methods between the devices with the same technology (such as WiFi/ZigBee) are to isolate spectrum across different time, frequency, and space. Examples of coarse frequency-based isolation include [9,
10, 12, 15, 16]. Examples of fine-grained frequency-based fragmentation include [11, 13, 17-19]. Several approaches use time-domain isolation based on centralized scheduling or distributed carrier senses such as $[20,21]$. LTE uses space division method to isolate the interference between different devices. The spectrum sharing between homogeneous technologies is easy to manage since they have the same $\mathrm{PHY} / \mathrm{MAC}$ protocols. Compared with current homogeneous technologies spectrum sharing methods, our method focuses on cross technologies spectrum sharing for optimizing the spectrum utilization in $5 \mathrm{GHz}$ unlicensed bands.

(ii) Spectrum Sharing between Heterogeneous Technologies. TIMO [22] is the pioneer work to manage the spectrum between cross technologies (WiFi and other technologies in $2.4 \mathrm{GHz}$ ); TIMO uses nulling to let the interferer decode its signal. ZIMO [23] decodes both WiFi and ZigBee signals. However, in $[22,23]$ the ZigBee and cordless phone occupy a much narrower channel and have lower power than WiFi signals; in this paper we propose solving the spectrum sharing problem between LTE and WiFi with the similar bandwidth and power.

(iii) Spectrum Sharing between LTE and WiFi in Unlicensed Bands. The current works related to the coexistence of $\mathrm{WiFi}$ and LTE are classified into three categories, LTE Unlicensed (LTE-U) based work, License-Assisted Access (LAA) based work, and simultaneously transmission work.

LTE-U was firstly proposed by Qualcomm and maintained by LTE forum [24]; the main principle of LTE-U is isolating the access of LTE and WiFi devices by the duty cycle of LTE. Reference [25] analyzed the performance of coexistence of LTE and WiFi by which WiFi allocated the channel when the duty cycle of LTE is in the "off" status; the results show that LTE-U based methods are unfair for WiFi devices. To improve LTE-U method, [26-30] make a more careful design of LTE-U to increase the throughput of WiFi devices. Reference [31] proposed a novel distributed algorithm for opportunistic sharing of unlicensed bands among LTE and WiFi devices (more details related to LTE$\mathrm{U}$ are discussed in Section 3).

LAA was proposed and maintained by $3 \mathrm{GPP}$ as the first standard to achieve the coexistence of WiFi and LTE technologies; the main principle of LAA is introducing carrier sensing mechanism to LTE PHY layer [8]. Reference [32] analyzed the downlink performance of LAA with a simple Listen-Before-Talk mechanism and [33] tested the throughput of LAA based schema. The most original LAA methods are described in $[6,7,34,35]$. Channel switch is considered in LAA to achieve a harmonious coexistence with WiFi in [36-38] which investigated the energy efficiency of LAA system. References $[39,40]$ designed and implemented LAA system in practical (more details related to LAA are discussed in Section 3).

However, LTE-U and LAA based schema are all time division spectrum sharing methods preventing the further improvement of the spectrum utilization.

To further optimize the spectrum utilization between LTE and WiFi devices, [41, 42] proposed enabling the 


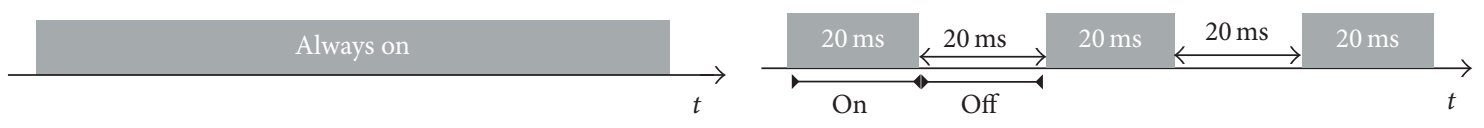

(a) Licensed LTE channel allocation

(b) LTE-U channel allocation



(c) LAA channel utilization

FIGURE 1: Current LTE and WiFi coexistence methods. (a) shows the channel utilization of LTE in licensed bands; LTE is a kind of scheduled networks in licensed bands; therefore, LTE always allocates the channel if there exists mobile traffic; (b) shows the channel utilization of LTE-U, which uses duty cycle to free channel to WiFi in fixed time domain; (c) shows the channel utilization of LAA, in which LTE shares the channel with WiFi by using Listen-Before-Talk (LBT) mechanism.

simultaneous transmission between LTE and WiFi coexisting networks. Reference [41] could enable the simultaneous transmission between LTE devices while our work aims to enable the simultaneous transmission between LTE and WiFi devices; the smartphone could receive he LTE and WiFi signals simultaneously by using the method proposed in [42]; however, it could receive the signals from only one LTE base station and WiFi access point from downlink. Our work aims to solve both the downlink and uplink problems with multiple LTE and WiFi devices.

\section{Background}

To achieve the coexistence of LTE and WiFi in $5 \mathrm{GHz}$ with efficient spectrum utilization, LTE forum and 3GPP propose using LTE Unlicensed (LTE-U) and LTE License-Assisted Access (LAA) separately. Most of the work introduced in Section 2 is LTE-U or LAA based work. In this section, we will describe the principles of LTE-U and LAA briefly and explain how to further improve the spectrum utilization by our method.

The main differences among traditional Licensed LTE, LTE-U, and LAA are described in Figure 1. Their main principles and differences are detailed as follows.

(i) Licensed LTE channel allocation: traditional Licensed LTE occupies the channel all the time if there is mobile traffic to be delivered. There are no other cross technologies such as WiFi or ZigBee sharing the same channel with LTE in the traditional licensed bands of LTE; therefore, Keep LTE in the "on" status for data delivery. However, offloading the traffic to unlicensed bands is avoidable since the licensed bands are scarce resource which is hard for managing the increasing global traffic (detailed in Figure 1(a)).

(ii) LTE-U channel allocation: LTE Unlicensed was first proposed by Qualcomm and promoted by LTE-U forum. LTE-U introduces duty cycle to coordinate the channel utilization between LTE and WiFi devices. Figure 1(b) is a simple example showing how LTE shares the channel with WiFi devices when LTE duty cycle is $50 \%$. In Figure 1(b), LTE allocates and frees the channel during every $20 \mathrm{~ms}$ alternatively. Therefore,
WiFi could allocate the channel when LTE is in the status of "off."

(iii) LAA channel allocation: LAA was proposed by 3GPP. LAA introduces Listen-Before-Talk (LBT) to coordinate the channel utilization between WiFi and LTE devices. Figure 1(c) shows that LTE devices listen to the channel first before sending or receiving the data. Therefore, both WiFi and LTE devices could allocate the channel to sending data if the channel is idle.

In summary, both LTE-U and LAA are time division multiple access-like (TDMA-like) schedule method for channel utilization between LTE and WiFi devices though they defined different channel management methods (detailed in Figures 2(a) and 2(b)). LTE-U and LAA could achieve the coexistence of WiFi and LTE. However, the TDMAlike channel allocation methods introduce transmission time delay; therefore, it is urgent to find new channel management methods to further improve the channel utilizations. In this paper, we propose using the parallel channel utilization method (detailed in Figure 2(c)) to optimize the channel utilization between $\mathrm{WiFi}$ and LTE devices in $5 \mathrm{GHz}$ unlicensed bands.

\section{Motivation}

How to solve the conflicts is the key issue to achieve coexistence of LTE and WiFi. Previous studies focus on avoiding these conflicts, such as LTE-U schedules WiFi frames with the duty cycle of LTE, and LAA adds LBT mechanism to avoid conflicts with WiFi. In summary, both of them assume that LTE and WiFi should allocate the channel in different time slots to avoid conflicts. However, time division utilization hardly achieves further improvement of spectrum efficiency and it also would cause time delay especially in dense deployment scenarios. To enable LTE and WiFi coexisting with lower transmission delay and higher spectrum efficiency, this section initializes a new conflict dealing method to motivate efficient channel allocation method in LTE and WiFi coexisting networks. In order to describe this easily, some definitions used in the remaining parts of this paper are defined in Abbreviations.

4.1. The Need for New Conflict Resolving Method. Spectrum utilization efficiency would be significantly improved if we are 




(a) LTE-U

(b) LAA

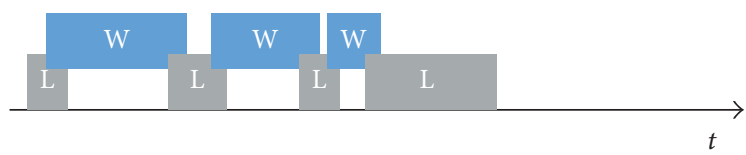

(c) Our method

FIGURE 2: Channel utilization of our method, LTE-U and LAA. LTE-U and LAA are TDMA-like channel allocation methods which can not further improve channel utilization efficiency; our method introduces parallel transmission method to enable the concurrent transmission between WiFi and LTE devices.

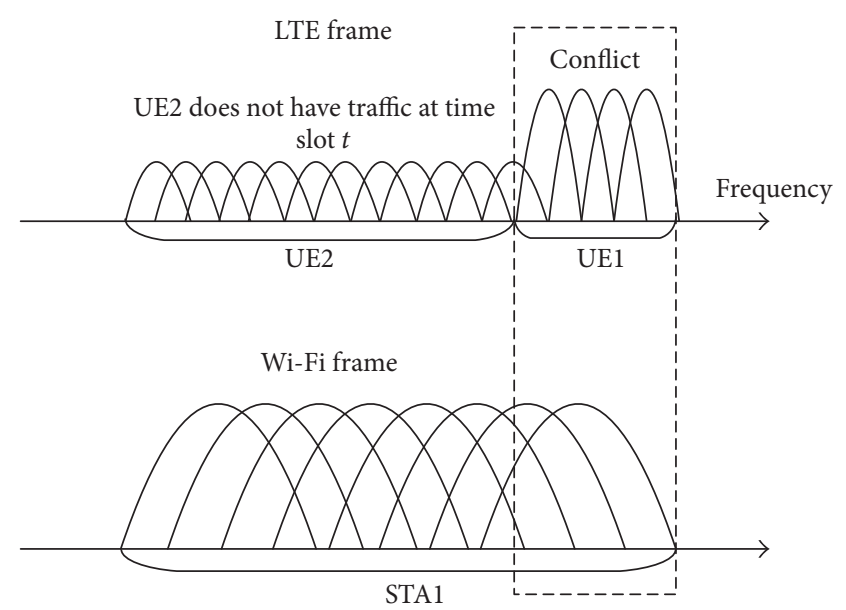

(a) Conflicts between LTE and WiFi when they coexisted

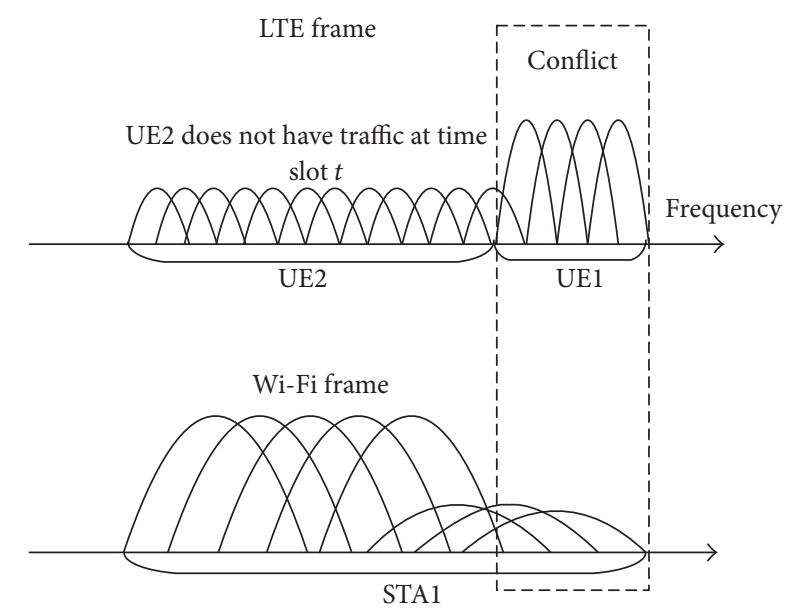

(b) Conflict resolving method by disabling some subcarriers

FIGURE 3: Conflicts in LTE and WiFi coexisting networks and a promising conflict resolving method by disabling some subcarriers of WiFi frames.

able to enable LTE and WiFi transmit simultaneously in the same channel. As we know WiFi APs only communicate with a single client in one time slot while LTE could communicate with multiclients by using Orthogonal Frequency-Division Multiple Access (OFDMA) method. A simple example shown in Figure 3(a) introduces this difference. When eNB connects with client UE1 and client UE2, AP connects with client STA1 only. But only UE1 and STA1 are in the conflict region; therefore, UE2 could receive the message correctly while UE1 and STA1 are certain to encounter the conflicts if they are transmitting in the same time slot. However, the spectrum utilization efficiency would significantly improve if LTE and WiFi transmit simultaneously and the data could recover from the conflicts.

One of the promising solutions which could make the data survive from the conflicts is shown in Figure 3(b). If the subcarriers in WiFi which interfere with STA1 are disabled, then the data transmitted UE2 could survive from the conflicts while the remaining subcarriers of WiFi which are not disabled also could survive. In order to evaluate how much spectrum efficiency would be increased by this subcarrier disabling method, we do a simple one AP and one eNodeB experiment, with the details in the Observation.
Observation. Spectrum utilization efficiency of parallel channel utilization schema is better than traditional time division method.

Experiment Setup and Results. One eNodeB with multiclients is deployed firstly; then, we deploy one eNodeB with multiclients in the conflict region with eNodeB. We think about an extreme situation that each client want to send message in every time slot for both LTE and WiFi.

The spectrum efficiency we observed is shown in Figure 4. If we use the subcarrier disabling method as channel parallel utilization schema, we can figure out that by utilizing this schema the spectrum utilization efficiency of this coexisting network is 1.6 times that utilizing time division schema.

4.2. The Need for New Channel Allocation Method to Achieve Efficient Spectrum Utilization in $5 \mathrm{GHz}$. Besides the channel allocation mechanisms, many works should be researched to achieve the coexistence of WiFi and LTE networks, such as network architecture, protocol design, and QoS balancing. However, as a basic technology, channel allocation mechanism is an essential part to achieve high efficiency coexistence of cross technologies.

Since $2.4 \mathrm{GHz}$ unlicensed band is too crowded, we should enable LTE and WiFi coexisting in $5 \mathrm{GHz}$; the new features of 


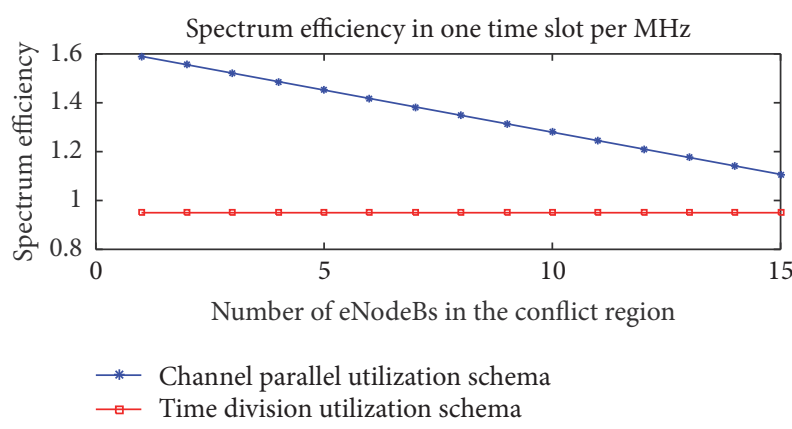

FIGURE 4: Spectrum efficiencies of time division channel utilization method and parallel channel utilization method.

the channels in $5 \mathrm{GHz}$ cause new challenge for allocation as follows.

(i) Nonoverlapped Channel Used in $5 \mathrm{GHz}$ for the Dense Deployment. Until this paper, there are only 9 nonoverlapped $20 \mathrm{MHz}$ channels used in $5 \mathrm{GHz}$ in United States. The nonoverlapped feature in $5 \mathrm{GHz}$ is much different from the overlapped channel in $2.4 \mathrm{GHz}$. Clients would face a serious conflict once they can not choose an exclusive channel especially in dense deployment scenario. If LTE is offloading their traffic in $5 \mathrm{GHz}$, the scarcity of these nonoverlapped channels would be more obvious.

(ii) Most Operators Want to Deploy eNodeBs and Get a Full Coverage in the Deployed Scenarios. These competitions between operators make the nonoverlapped channel in $5 \mathrm{GHz}$ more scarce, because we should assign different channels to each operator for avoiding interference interoperators. As far as we know, there are six main operators in United States that provide LTE services. They are AT\&T, Claro, T-Mobile, Sprint, Open Mobile, and Choice Communications. From the competition view, each company wants to offload their traffic into $5 \mathrm{GHz}$, which makes the nonoverlapped $20 \mathrm{MHz}$ channel get more inefficient.

(iii) Different Media Access Methods of LTE and WiFi Networks Make the Negotiation between LTE and WiFi More Difficult. Carrier Sensing Media Access (CSMA) was used in WiFi network to schedule channel access, while LTE utilizes Orthogonal Frequency-Division Multiple Access (OFDMA) to schedule different resource belonging to different clients.

In summary, new channel allocation mechanism is urgent for LTE and WiFi coexisting networks to manage the spectrum utilization. To fill this gap, in this paper we present Conflict-Tolerant Channel Allocation (CTCA) (in Section 4) to achieve efficient spectrum utilization.

\section{Preliminaries}

This section introduces the assumptions and the network model related to the rest of our work.

5.1. Assumptions. The assumptions behind our works are as follows.
(1) The WiFi networks are isomorphic, running 802.11ac protocols in $5 \mathrm{GHz}$ band, the channel width is $20 \mathrm{MHz}$, and they only use 9 channels in $5 \mathrm{GHz}$. This assumption is reasonable because in US only 9 channels are used in $5 \mathrm{GHz}$ for WiFi network. Our work aims to resolve channel sharing problem among these 9 channels while it is also easy extend to other channels.

(2) The eNodeBs from different operators deployed Pico nodes in $5 \mathrm{GHz}$. We assume Picos could get the location information of its clients. This assumption is also reasonable because LTE can recognize the clients in the edge for handover. We could just use this function to get the clients location information.

(3) eNodeBs from different operators allocate different channels to avoid interferences. Definitely, there may exist some mechanisms to avoid the conflicts between eNodeBs from different operators when they share the same channel, but it is out of our research scope in this paper.

In LTE and WiFi coexisting networks, there are more conflicts when AP and eNodeB allocate the same channel. However, the current time division channel utilization schema is limited for further efficient spectrum utilization and also caused a transmission delay. To resolve this problem, in Section 4, we propose Low Amplitude Stream Injection (LASI) to deal with these conflicts and motivate a new ConflictTolerant Channel Allocation (CTCA) method to increase the spectrum utilization and guarantee the transmission time delay in LTE and WiFi coexisting network.

5.2. Network Model. Under these assumptions, we define the network model. Suppose most of the operators want to deploy their eNodeBs and provide LTE service in a dense deployment scenario such as a stadium or music concert with thousands of audiences. WiFi service providers also want to deploy access points in these scenarios. The network architecture of LTE and WiFi coexisting networks is as shown in Figure 5, where $n$ represents the number of WiFi access points in the network model; that is, $\mathrm{AP}=\left\{\mathrm{AP}_{1}, \mathrm{AP}_{2}, \ldots, \mathrm{AP}_{n}\right\} . m$ represents the number of eNodeBs from different operators. We assume that different operators utilize different channels to avoid interference in assumption (3). Therefore, it does not need to distinguish eNodeBs from different operators; the total number of eNodeBs is $\mathrm{eNB}=\left\{\mathrm{eNB}_{1}, \mathrm{eNB}_{2}, \ldots, \mathrm{eNB}_{m}\right\}$.

We suppose there are multiclients connected with $\mathrm{AP}_{i}$, and we define client $j$ connected to $\mathrm{AP}_{i}$ as $\mathrm{STA}_{i j}$. Similarly, we also define $\mathrm{UE}_{i j}$ as client $j$ which connects to $\mathrm{eNB}_{i}$.

\section{Main Design}

Depending on the network model defined in Section 5, the overall design of our approach is presented in this section. We first propose Low Amplitude Stream Injection (LASI) method to enable data recovery from conflicts. To further utilize the LASI method, we introduce the Conflict-Tolerant Channel Allocation (CTCA) and its detailed steps secondly. 


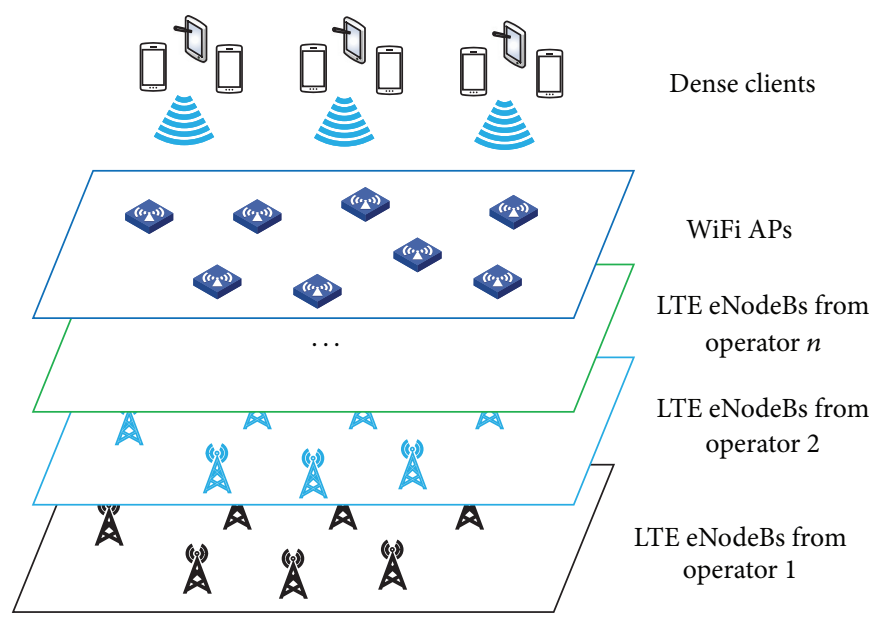

FIgURE 5: Architecture of LTE and WiFi coexisting network in $5 \mathrm{GHz}$ band.

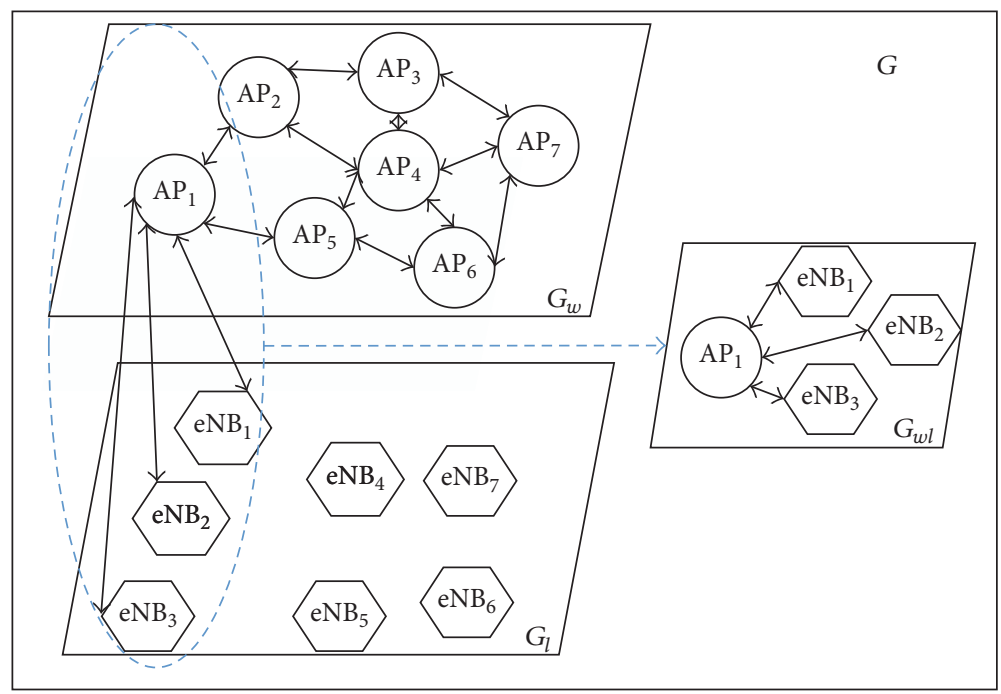

FIGURE 6: Multigraph model of LTE and WiFi coexisting network in $5 \mathrm{GHz}$.

6.1. Design Overview. Based on the network model, eNodeBs would get a fixed channel first while eNodeBs from the same operator share the fixed channel. APs would allocate channels in three steps: (i) choosing an idle channel which has not been allocated by neighbor nodes (including APs and eNBs); (ii) choosing a channel which is allocated by neighbor APs and sharing the channel with time division schema; and (iii) choosing a channel which is allocated by the neighbor eNodeBs from one operator.

Due to the different types of nodes (APs and eNodeBs), we model this problem as a multigraph coloring problem. Graph $G=G_{l} \cup G_{w} \cup G_{w l}$. The relationship of these three subgraphs is shown in Figure 6, where $G_{l}=\left(V_{l}, E_{l}\right)$ represents the conflicts in LTE network. In subgraph $G_{l}$, $V_{l}=\left\{\mathrm{eNB}_{1}, \mathrm{eNB}_{2}, \ldots, \mathrm{eNB}_{m}\right\}$, and $E_{l}=$ NULL since we do not consider the interference between eNodeBs in this paper (see assumption (3)). $G_{w}=\left(V_{w}, E_{w}\right)$ represents the conflicts in WiFi networks. In subgraph $G_{w}, V_{w}=\left\{\mathrm{AP}_{i} \mid i \epsilon\right.$ $(1,2, \ldots, n)\}$, and we add an edge between $\mathrm{AP}_{i}$ and $\mathrm{AP}_{j}$ if they have potential to interfere with each other. $G_{w l}=\left(V, E_{w l}\right)$ represents the conflict between LTE and WiFi networks. In subgraphs $G_{w l}, V=V_{l} \cup V_{w}$, and we add an edge between $\mathrm{AP}_{i}$ and $\mathrm{eNB}_{j}$ if they have potential to interfere with each other.

There are two design goals of Conflict-Tolerant Channel Allocation (CTCA) method presented in this section: (i) optimizing spectrum utilization and (ii) decreasing network latency in this coexisting networks. To achieve the first design goal, AP should first allocate idle channels for information transmission and decide which channel to allocate once no idle channel left. To achieve the second design goal, AP should try best to avoid time division channel access method. If there exist cochannel utilization situations between APs and eNodeBs, we explore LASI method to enable data to recover from conflicts. In summary the overall design is shown in Figure 7.

The CTCA in Figure 7 consists of three parts; the first part is LASI method shown in Figure $7(\mathrm{a})$. The second part is nonoverlapped channel allocation in $G_{w}$ shown in 

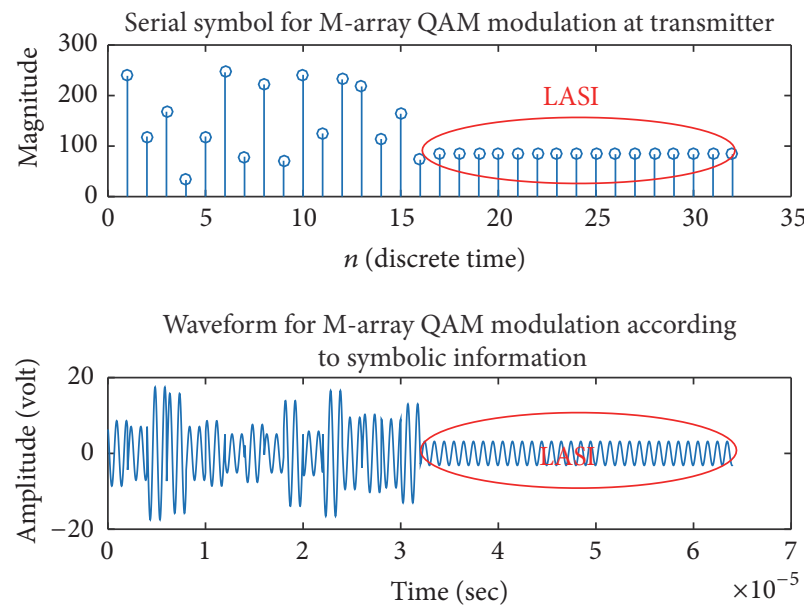

(a) Low Amplitude Stream Injection (LASI) method

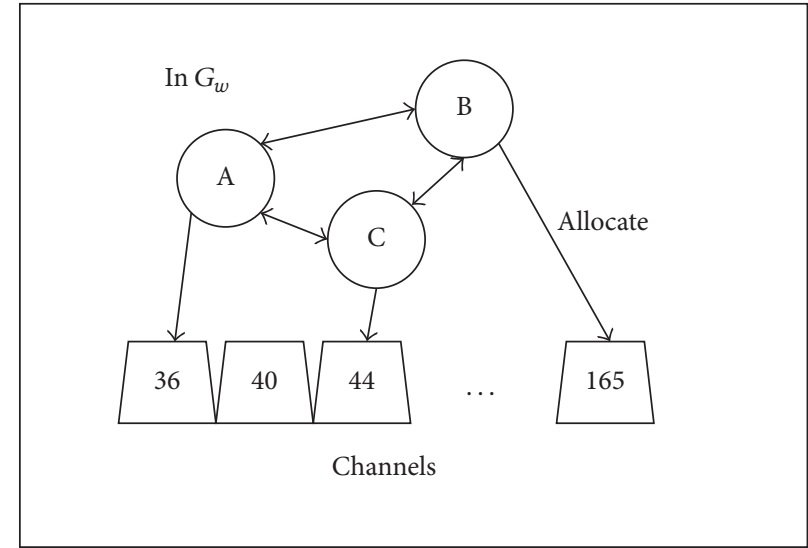

(b) No-overlap channel allocation

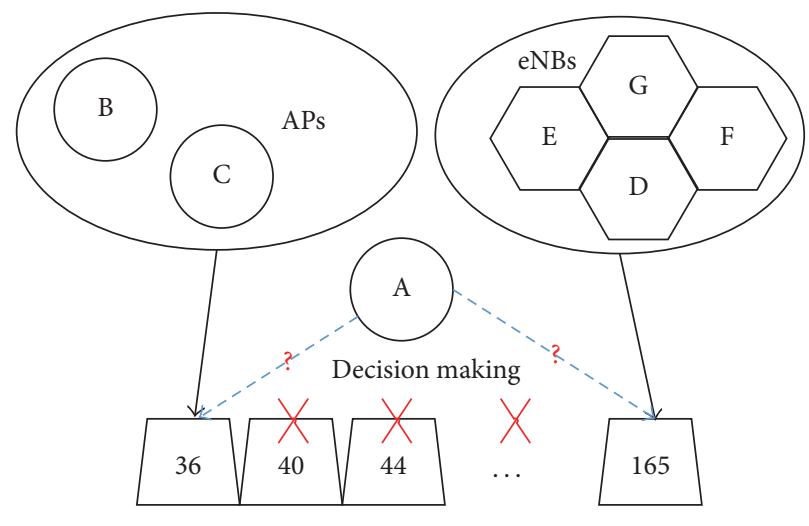

Channels

(c) Cochannel allocation decision making

Figure 7: Design overview of Conflict-Tolerant Channel Allocation (CTCA) method.

Figure $7(\mathrm{~b})$. The last part is the cochannel allocation decision making part shown in Figure 7(c).

6.1.1. LASI Conflicts Resolving Method. LASI shown in Figure 7(a) is applied for resolving conflicts between APs and eNodeBs in LTE and WiFi coexisting networks. LASI is a practical implementation of subcarrier disabling method (see Section 2) to achieve channel parallel utilization. LASI method makes data recover from the conflicts. The details of LASI will be introduced in Section 6.2.

6.1.2. Nonoverlapped Channel Allocation in $G_{w}$. APs apply this step to allocate an idle channel from the remaining channels after eNodeBs finishing their fixed channels. Each AP allocates the channel and optimizes the conflict to zero (i.e., choose different channels from their neighbors). If no such channels left, jump to the cochannel allocation decision making part.

6.1.3. Cochannel Allocation Decision Making in G. APs apply this step to decide which channel to utilize once there are no idle channels to choose according to their neighbors. In this paper, we collect the client information of the neighboring nodes for this decision making. The details of this method will be introduced in Section 6.3.

By applying these three steps, CTCA algorithm enables LTE and WiFi to allocate proper channels for increasing spectrum utilization and decreasing network latency.

6.2. LASI Conflict Resolving Method. We first present the Low Amplitude Stream Injection (LASI) method in this section. secondly we proved the correctness of LASI and also discussed the additional delay by utilizing LASI method in LTE and WiFi network.

6.2.1. Procedure of LASI. LASI is proposed to resolve conflicts between LTE and WiFi frames in MAC layer. LASI utilizes both the features of OFDMA in LTE and CSMA in WiFi network. As we described in Figure 3 (see Section 2), we can protect both part of WiFi frame and some LTE subframes (e.g., subframes belonging to UE2) by disabling some subcarriers in the conflict region.

However, disabling some subcarriers is hard to implement; therefore we propose the practical Low Amplitude Stream Injection method, short for LASI method to enable data to recover from conflicts. LASI injects some lower 


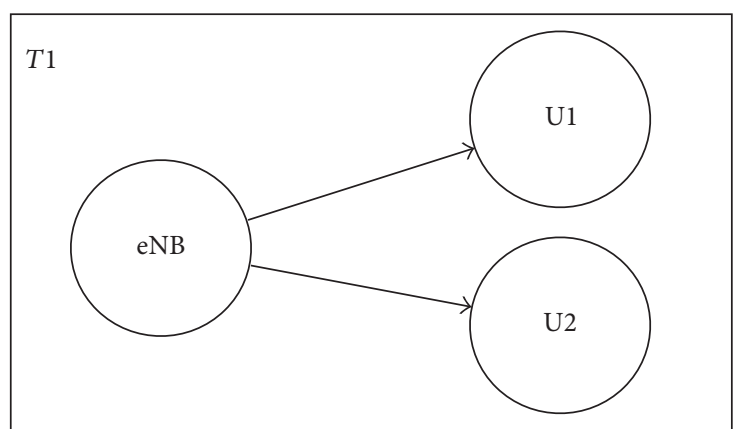

(a) T1: eNodeB sense clients in conflict region

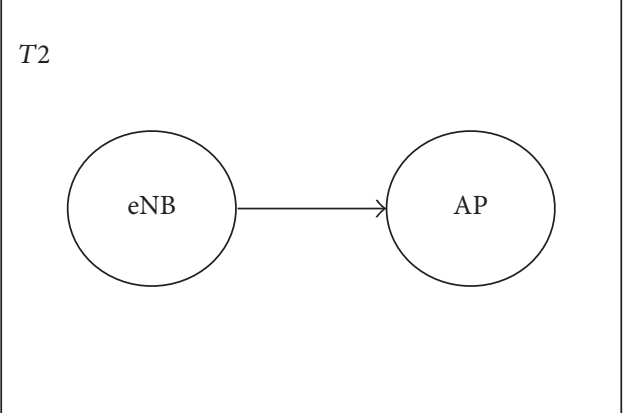

(b) T2: eNodeB exchange injected stream information to AP

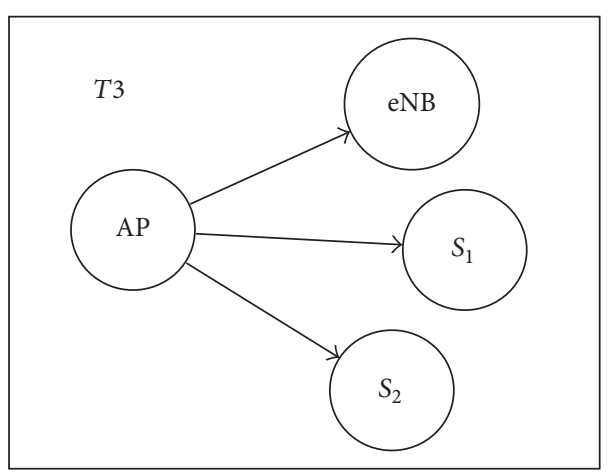

(c) AP notifies its client and eNodeB about the injected stream information

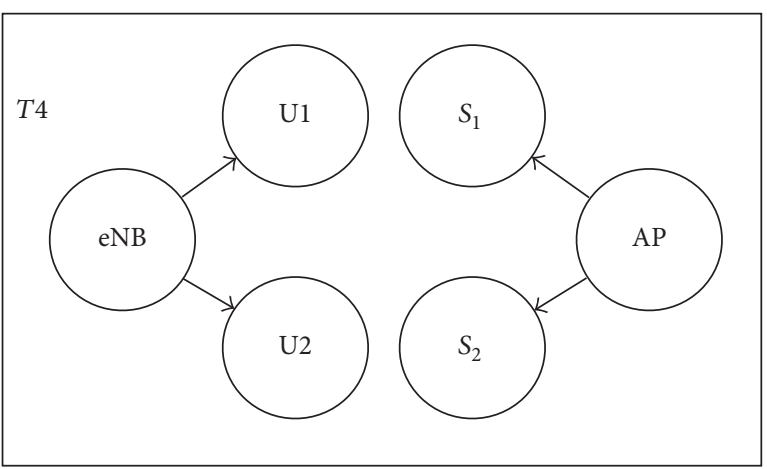

(d) eNodeB and WiFi begin to communicate with their clients

FIGURE 8: Negotiation and synchronization procedure for LTE and WiFi to communicate with subcarrier to be injected by Low Amplitude Stream.

amplitude stream in the subcarriers of WiFi frames which affects LTE clients in the conflict region. Figure 7(a) introduces the key principles of LASI. LASI could be easily implemented by quadrature amplitude modulation (QAM).

In order to negotiate which and how many subcarriers should be injected in WiFi frames, LTE should exchange control information with the APs sharing the same channel. The negotiation procedure is shown in Figure 8. From Figure 8 , four time slots are needed to negotiate which and how many subcarriers to be injected into WiFi frames. The details of the four steps are as follows.

(i) T1: eNodeB first senses the clients in conflict region. Figure $8(\mathrm{a})$ describes this activity of time slot $T 1$. If eNodeB sensed some clients in conflict region, procedure goes to $T 2$.

(ii) T2: eNodeB tells WiFi access point which and how many subcarriers should be injected by Low Amplitude Stream to avoid the conflicts between eNodeBs in the conflict region (see Figure 8(b)).

(iii) T3: WiFi broadcasts the injected stream information to its clients and the eNodeB that sent the information in T2 (see Figure $8(\mathrm{c})$ ). Therefore, the WiFi clients could decode the information correctly, and eNodeBs also could get ready to exchange messages with their clients. (iv) T4: WiFi access point and LTE transmit information to their own clients, respectively (see Figure 8(d)). This is the normal transmission after the negotiation.

6.2.2. Theoretical Analysis. We prove the correctness of LASI and also analyze the latency caused by LASI method.

Theorem 1. LASI is correct from the theoretical level by utilizing quadrature amplitude modulation (QAM) and orthogonal frequency-division multiplexing (OFDM) technology.

Proof. Consider that a conflict occurs between LTE frame and WiFi frame in the same channel. We define the signal $S_{1}$ in physical layer of LTE frame in (1), and we also define the signals of WiFi frame as $S_{2}$ in (2):

$$
\begin{aligned}
& S_{1}=\sum_{j 1=1}^{m 1} a_{j 1} * f_{i}+\sum_{j 2=m 1}^{m 2} a_{j 2} * f_{i}+\sum_{j 3=m 3}^{m} a_{j 3} * f_{i}, \\
& S_{2}=\sum_{i 1=1}^{n 1} A_{i 1} * F_{i}+\sum_{i 2=n 1}^{n 2} A_{i 2} * F_{i}+\sum_{i 3=n 2}^{n} A_{i 3} * F_{i} .
\end{aligned}
$$

In (1) and (2), we assume that $\sum_{i 2=n 1}^{n 2} A_{i 1} * F_{i}$ and $\sum_{j 2=m 1}^{m 2} a_{j 1} * f_{i}$ are in the conflict region. If we inject the lowest amplitude 
stream in case the modulation is 256 QAM, the amplitude of the injected stream is calculated by the formula

$$
\underset{\forall k \in\left[j_{2} \rightarrow m_{2}\right]}{a_{k}}=|(1 \pm i)| \approx 1.41,
$$

where $a_{k}$ is $63 \%$ of the second lowest amplitude and only eight percent of the highest amplitude; thus the injected stream could avoid the conflicts in a promising degree. Besides this $a_{k}\left(k \in\left(j_{2}\right.\right.$ to $\left.\left.m_{2}\right)\right)$ are equal and in the same frequency which almost could not affect other signals transmitting in the same channel. Thus LSAI is correct from the theoretical level.

Theorem 2. There exists 1 time unit delay when one pair of eNodeBs and APs are running LASI method.

Proof. In T1, eNodeBs always sense their clients and calculate the locations of the clients; thus the first $T 1$ phase does not introduce additional time delay. In T2, eNodeB should send a notification to AP; this introduces an additional time delay. For T3, WiFi always uses CSMA to sense channels before they transmit; we just utilize this CSMA carrier to notify the client and eNB about the information of injected subcarriers; therefore $T 3$ is not the additional time delay, so T3 does not introduce additional time delay. In $T 4$, it also does not introduce additional time delay because it is a normal transmission between eNodeB/AP and their clients.

6.3. Nonoverlapped Channel Allocation in $G_{w}$. APs first allocate channels from the remaining ones when eNodeBs finish their channels setting. Allocating the remaining nonoverlapped channel could minimize the conflicts in the network. this section introduces the nonoverlapped channel allocation algorithm first followed by its theoretical analysis.

6.3.1. Nonoverlapped Channel Allocation Algorithm. $G_{w}$ presents the graph of WiFi network; in order to minimize the conflicts, WiFi APs first allocate the nonoverlapped channels with $20 \mathrm{MHz}$ width after eNodeBs had allocated their channels. The nonoverlapped channel assignment problem for WiFi network can be modeled as a graph coloring problem of graph $G_{w}$ in which the APs are the vertices of a graph. A conflict between two APs is represented by an edge in the graph. The goal of this graph coloring problem is to assign a set of distinct colors. To enable the most efficient use of these channels the objective of this issue is to color graph $G_{w}$ with minimum number of colors and avoid any conflicts.

The nonoverlapped channel allocation problem in $G_{w}$ is stated as follows. A channel assignment $C\left(\mathrm{AP}_{i}\right), \mathrm{AP}_{i} \in V_{w}$, is a mapping $C: V_{w} \rightarrow\{1,2, \ldots, k\}$ from the set of vertices to the set of colors. We say that an edge $\left(\mathrm{AP}_{i}, \mathrm{AP}_{j}\right)$ is conflictfree edge when $\mathrm{AP}_{i}$ and $\mathrm{AP}_{j}$ allocate different nonoverlapped channel (all the $20 \mathrm{MHz}$ channels in $5 \mathrm{GHz}$ used in United States are nonoverlapped channels); otherwise if they share the same channel we say edge $\left(\mathrm{AP}_{i}, \mathrm{AP}_{j}\right)$ is a conflict edge.

The interference of $\mathrm{AP}_{i}$ and $\mathrm{AP}_{j}$ is serious once they share the same channel. Since there is no overlapped channel in $5 \mathrm{GHz}$, if the conflict occurs, it occurs on the whole channel, not partially described in [13]. So in order to minimum the conflicts between APs in $G_{w}$, we define conflict factor of WiFi network as $C F_{w}$, and $C F_{w}$ is presented as

$$
C F_{w}\left(\mathrm{AP}_{i}, \mathrm{AP}_{j}, c\right)= \begin{cases}0, & \left(\mathrm{C}\left(\mathrm{AP}_{i}\right) \neq C\left(\mathrm{AP}_{j}\right)\right) \\ 1, & \left(\mathrm{C}\left(\mathrm{AP}_{i}\right)=C\left(\mathrm{AP}_{j}\right)\right)\end{cases}
$$

$C F_{w}\left(\mathrm{AP}_{i}, \mathrm{AP}_{j}, c\right)$ presents the total effect of conflict in an edge, $c$ represents the color of node $\mathrm{AP}_{j}$, and the objective in this phase is to optimize the conflicts between APs. If we can not achieve this goal, we just let the APs try the third phase in Section 6.4. So the objective in this phase is shown as (5) when we find a mapping $C$ for graph $G_{w}$.

$$
\max \sum_{\forall e=\left(\mathrm{AP}_{i}, \mathrm{AP}_{j}\right) \in E_{w}} C F_{w}\left(\mathrm{AP}_{i}, \mathrm{AP}_{j}\right)=0 .
$$

6.3.2. The Detailed Algorithm. The nonoverlapped channel allocation algorithm in $G_{w}$ is shown as Algorithm 1, which has two steps according to the algorithm.

Initialization Step. Line (1) to Line (7) introduce the initialization step; in Line (2), all the APs points are assigned an initial color, and in Line (5)CF $\left.\mathrm{CP}_{w}, \mathrm{AP}_{j}\right) \in E_{w}$ was initialized.

Optimization Step. In this step, we begin to optimize the conflicts in Lines (9), (10), (11), (12), and (13). The objective functions are shown in (1), and we should make sure the value of this function is zero; if we can not guarantee this value the algorithm breaks in Line (15) and is called cochannel allocation algorithm proposed in Section 6.4.

6.3.3. Complexity Analysis. In assumption (1) we assume that we discuss the channel reuse in $5 \mathrm{GHz}$ and share the 9 nonoverlapped WiFi channels with a width of $20 \mathrm{MHz}$. Thus the maximum number of the colors is 9; therefore $C=$ $1,2, \ldots, 9$. We also assume that the network is connected when in dense deployment.

So the complexity of nonoverlapped channel allocation part depends on the number of reminding channels after LTE allocate the channels. The details are analyzed below. We assume that $k$ is the remaining channel, and we have the following theorems.

Theorem 3. Nonoverlapped channel allocation is polynomial time solvable problem when $k \leq 2$.

Proof. Given a graph $G=(V, E),(1)$ coloring graphs are the empty graphs and (2) coloring problem is equivalent to bipartite graph determining problem. We divided $V$ into two disjoint sets $V_{1}$ and $V_{2}$; that is, $V_{1}$ and $V_{2}$ are independent sets, and every edge connects a vertex in $V_{1}$ to one in $V_{2}$, we assume $\left|V_{1}\right|=m$ and $\left|V_{2}\right|=n$, we set different colors to the vertexes connected by edge in sets $V_{1}$ and $V_{2}$, and the complexity is $O(m \times n)$, which is polynomial time solvable problem.

Theorem 4. Nonoverlapped channel allocation is an NP-hard problem when $k \geq 3$. 


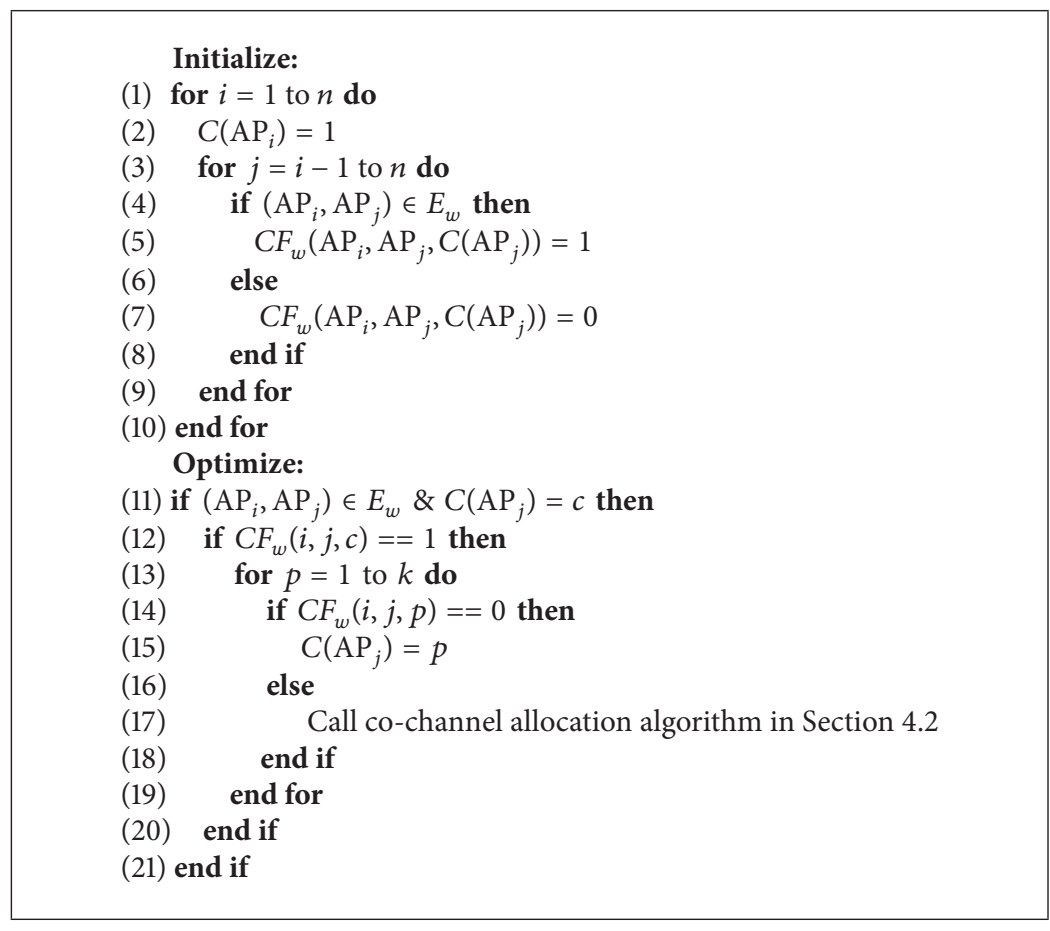

Algorithm 1: Nonoverlapped channel allocation in $G_{w}$.

Proof. Consider the general problem of coloring an undirected graph: given a graph $G=(V, E)$, does there exist $k$ coloring $C(k \geq 3): v \rightarrow\{1 \cdots k\}, 1 \leq k \leq|V|$, such that $\forall e=\left(v_{i}, v_{j}\right) \in E \Rightarrow C\left(v_{i}\right) \neq C\left(v_{j}\right)$ ? We formulate our nonoverlapped channel allocation as this general problem with the objective function $\forall e=\left(\mathrm{AP}_{i}, \mathrm{AP}_{j}\right) \mathrm{CF}_{w}\left(\mathrm{AP}_{i}, \mathrm{AP}_{j}\right)=$ 0 ; thus nonoverlapped channel allocation is an NP-hard problem since the general coloring problem is NP-hard problem.

6.4. Cochannel Allocation Decision Making in G. There may be some APs that did not allocate any channel after the nonoverlapped channel allocation phase since the design goal of nonoverlapped channel allocation is to optimize the conflicts in $G_{w}$; if there are no idle channels to allocate in $G_{w}$, the procedure would move to cochannel allocation decision part. The most important challenge in this part is how to decide which channel to allocate.

6.4.1. Parameters Design for Cochannel Allocation Decision Making. If there are no exclusive channels to allocate, AP has to share the same channel with other APs or other multieNodeBs from one operator, and these two types of decisions are analyzed as follows.

Type 1: Cochannel with Another AP. WiFi uses CSMA to isolate data transmission between different APs, which is a time division method, and the transmission time delay will also increase when waiting to allocate the channel.

Type 2: Cochannel with Multi-eNodeB from One Operator. More conflicts are confronted when APs share the same channel with eNodeBs. However, LASI method can resolve this kind of conflicts.

So, AP should make a decision whether to choose to have cochannel with another AP or multi-eNodeBs. And this selection should consider the spectrum efficiency and transmission time delay since we can utilize LASI method to solve conflicts between LTE and WiFi. For the cochannel decision between Type 1 and Type 2, we define $N_{c}(i)$ as a parameter to make this decision $N_{c}(i)$ present the number of clients that can be solved per time slot and per $\mathrm{MHz} . N_{c}(i)$ is calculated by

$$
\begin{aligned}
& N_{c}(i) \\
& =\frac{\sum_{\forall\left(\mathrm{AP}_{i} \in G_{w}\right) \& C\left(\mathrm{AP}_{i}\right)=c} n_{i}+\sum_{\forall\left(\mathrm{eNB}_{j} \in G_{w l}\right) \& C\left(\mathrm{eNB}_{j}\right)=c} N_{j}}{T * B_{w}},
\end{aligned}
$$

where $n_{i}$ presents the number of clients served by $\mathrm{AP}_{i}$ and $N_{j}$ represents the number of clients served by $\mathrm{eNB}_{j}$, while $T$ is the time we measured and $B_{w}$ is the width of the channels shared by AP and eNodeB. From (6), we can easily deduce the average number of clients that can be solved by two APs sharing the same channel in (7) and APs sharing the channel with multi-eNodeBs from a specific operator in (8).

$$
N_{w}(i)=\frac{N\left(\mathrm{AP}_{i}\right)+\sum_{j=1}^{m} N\left(\mathrm{AP}_{j}\right)}{B_{w} * t_{\text {slot }} * \sum_{j=1}^{m} N\left(\mathrm{AP}_{i}\right)},
$$




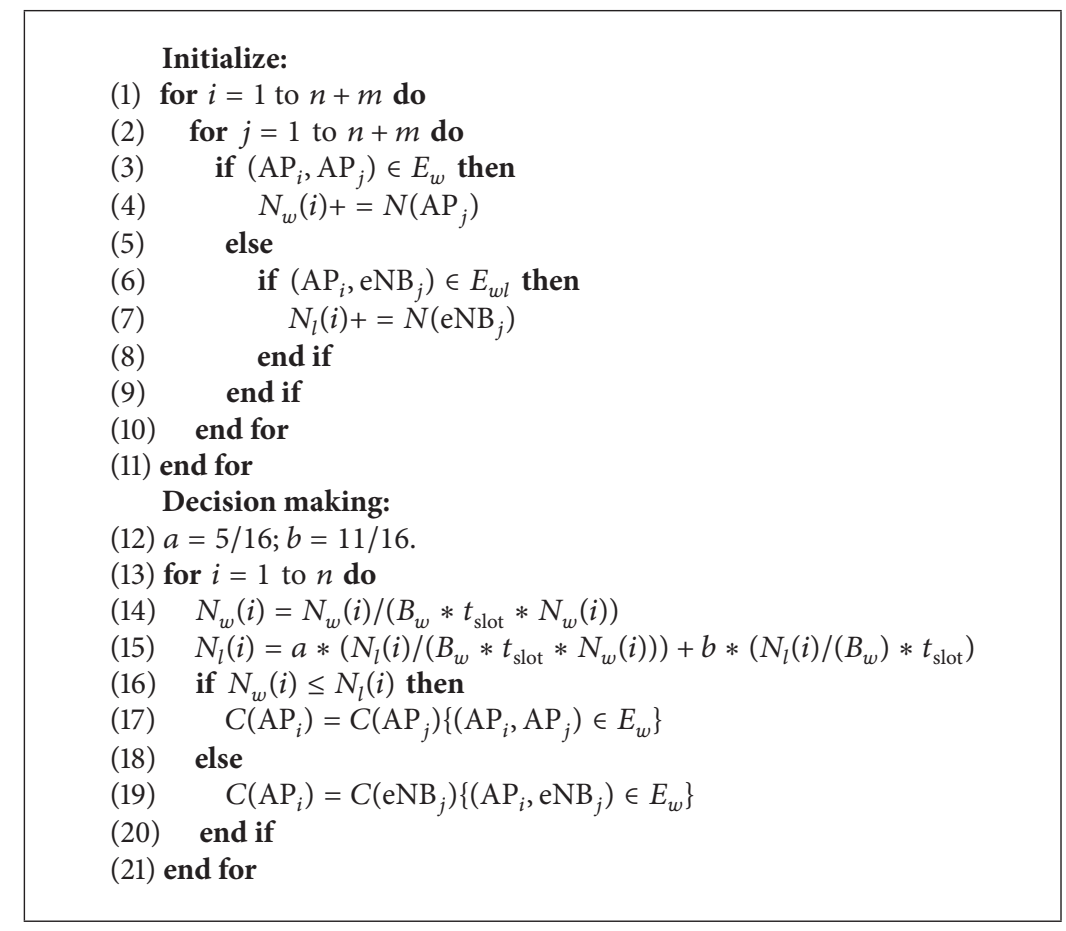

Algorithm 2: Cochannel allocation decision making algorithm in G.

$$
\begin{aligned}
N_{l}(i)=\alpha & * \frac{N\left(\mathrm{AP}_{i}\right)+\sum_{j=1}^{m} N\left(\mathrm{eNB}_{j}\right)}{t_{\text {slot }} * B_{w} * \sum_{i=1}^{m} N\left(\mathrm{eNB}_{j}\right)}+\beta \\
& * \frac{N\left(\mathrm{AP}_{i}\right)+\sum_{j=1}^{m} N\left(\mathrm{eNB}_{j}\right)-M}{t_{\text {slot }} * B_{w}} .
\end{aligned}
$$

6.4.2. The Detailed Algorithm. The cochannel allocation algorithm is shown as Algorithm 2. There are two phases in Algorithm 2, and the details are as follows.

Initialization. Line (1) to Line (10) are initialization phase; in this phase AP and eNode exchange the clients information with each other and utilize this information to calculate $N_{l}(i)$ and $N_{w}(i)$ in Line (4) and Line (7) separately.

Decision Making. AP makes the decision according to value of $N_{l}(i)$ and $N_{w}(i)$; if $N_{l}(i) \geq N_{w}(i), \mathrm{AP}_{i}$ choose to have cochannel with multi-eNodeBs; otherwise AP chooses to have cochannel with other APs.

6.4.3. Complexity Analysis of Cochannel Allocation Decision Making Algorithm. This section introduces the complexity of cochannel allocation decision making algorithm.

Theorem 5. The complexity of cochannel allocation decision making is $O(m * n)$.

Proof. Consider that in a graph $G=V, E$, finding a mapping $C: v_{i} \rightarrow 1 \cdots k(1 \leq k \leq|V|)$, the problem could be formulated as $\forall e=\left(v_{i}, v_{j}\right) \in E \Rightarrow C\left(v_{i}\right)=C\left(v_{j}\right)$; we assume $|V|=n$, and the complexity is $O(n)$. Cochannel allocation decision making algorithm is a two-step $v_{i} \rightarrow 1 \cdots k$. The first step is searching in $G_{w}=\left(V_{w}, E_{w}\right)$ and calculating the decision parameter $N_{w}(i)$, and secondly search in $G_{w} l=$ $\left(V_{w} l, E_{w} l\right)$ and calculate $N_{l}(i)$; we assume that $\left|V_{w}\right|=n$ and $\left|V_{w} l\right|$; thus the complexity of cochannel decision making problem is $O(m \times n)$.

\section{Evaluation}

We evaluate LTE and WiFi coexistence using multiple threads simulators which are implemented by Python. We set the channel bandwidth to $20 \mathrm{MHz}$, which is compatible to 802.11n/ac and LTE in licensed bands. LTE and WiFi allocate totally 8 channels in $5 \mathrm{GHz}$ (channels $36,40,44,48,149$, 153, 157, and 161). For WiFi, we implemented one kind of server (sender) and client (receiver) according to 802.1lac. For LTE, we implemented four kinds of servers and clients: LTE Control-free (LTE-C), LTE-U, LAA, and our approaches (CTCA); LTE-C, LTE-U, and LAA are three baselines; the main principles are detailed in Section 7.3.

7.1. Experiment Setup. We randomly deploy APs and LTE eNodeBs in a 200-meter square region, and we assume that the APs and eNodeB share the same transmission range of 35 meters, and the eNodeBs are Picos which provide indoor services. Six operators want to deploy their Picos in this region. The modulation of both APs and eNodeBs is QAM256, which is modulated by OFDM method. We fixed 36 APs and 36 eNodeBs in this region, the eNodeBs come from 6 operators, each operator deploys 6 eNodeB, and the eNodeBs from the same operator occupy the same channel (e.g., channel 48). The clients of the WiFi APs and LTE 
eNodeBs could be changed with different settings if required in the experiments. The transmission data rate is fixed, each operator gets fixed channel for transmission from other operators, and each AP should allocate a channel according to the standards defined in LTE-C (see Section 7.3), LAA, LTE$\mathrm{U}$, and CTCA methods.

7.2. Performance Metrics. To evaluate the efficiency of spectrum utilization when LTE and WiFi coexist in $5 \mathrm{GHz}$, we evaluate average transmission number, average transmission delay, and spectrum efficiency. The specific definitions are as follows.

Average Transmission Number. The average transmission number indicates how many packets could be transmitted/second/link (links include the WiFi links and LTE links). Therefore, the average transmission number could be calculated by

$$
A T N=\frac{\sum_{i=1}^{n} T N_{i}+\sum_{j=1}^{m} T N_{m}}{n+m},
$$

in which $A T N$ represents the average transmission number, $n$ represents $n \mathrm{WiFi}$ links, and $m$ represents $m$ LTE links. $T N_{i}$ and $T N_{j}$ represent the transmission number of WiFi link $i$ and LTE link $j$. Therefore, ATN * $(m+n)$ indicates the total throughput of the LTE-WiFi coexistence system.

Average Transmission Delay. Average transmission delay is the number of time units cost when transmitting one packet.

Spectrum Efficiency. Spectrum efficiency measured in this paper is defined as the ratio of transmission number and spectrum bandwidth.

7.3. Baseline. We compared our approach with the following baselines.

Baseline I: LTE Control-Free (LTE-C). LTE-C lets LTE and $\mathrm{WiFi}$ run in the same band without any channel management control. In LTE-C, LTE devices transmit as scheduled in the assigned channel, and WiFi devices utilize CSMA to access the channels when there is traffic to be resolved.

Baseline II: LTE-U. As described in Section 3, WiFi devices control their transmission by the duty cycle of LTE devices. WiFi devices transmit when the status of LTE devices is "off," while LTE devices transmit when the status is "on." Therefore, they use the channel alternatively but LTE has higher priorities.

Baseline III: LAA. LAA adds LBT mechanism to share the same channel with WiFi devices. In LAA method, both LTE and WiFi sense the channel before sending packets. The transmission delay of LTE and WiFi devices would be longer in dense deployed scenarios.

7.4. Performance Comparison. This section compares CTCA with LTE control-free (LTE-C), LTE Unlicensed (LTE-U), and License-Assisted access (LAA) methods. Average transmission number, average transmission delay, and spectrum efficiency are evaluated separately in this section.

7.4.1. Average Transmission Number. Firstly, the average transmission number was evaluated with different network parameters. And the results are shown as in Figures 9, 10, and 11.

Figure 9 describes the average transmission number of one link including WiFi and LTE links in a fixed transmission period with the change of the number of LTE clients. Figure 9(a) shows that the average transmission number decreases with the number of UE clients; the reason why we got the experimental results is that each individual LTE client could get less and less time slot for transmission with the increasing number of the total clients. However, the total transmission number increases in LTE-WiFi coexisting systems, and the average transmission number of CTCA increased by $24 \%$ of that LTE-C, $109 \%$, and $26 \%$ of LAA and LTE-U separately. As the number of UE clients increases, both WiFi and LTE devices will be "back off" since the ListenBefore-Talk mechanism. Therefore, the average transmission number of LAA method is the lowest one.

Figure 9(b) shows the average transmission number of LTE clients and WiFi clients separately. This kind of data could reveal some details of the LTE-WiFi coexistence system. We can see that CTCA and LTE-C algorithms could keep the average transmission number of LTE devices in a stable level with the increasing UE clients because LTE devices do not need to be back off when coexisting with WiFi devices, while the transmission numbers of LTE-U and LAA decrease sharply with the number of UE clients since the ListenBefore-Talk mechanism causes more conflict and back-off when the UE number increases.

Figure 9(b) also shows that the average transmission number of WiFi devices decreased with the number of UE clients. However, the average transmission number of our CTCA method is greater than those of LTE-C, LTE$\mathrm{U}$, and LAA since our method could utilize the channel simultaneously with LTE devices.

Figure 10 shows the trends of average transmission number with the increasing number of WiFi clients (STAs). With similar reason to the result in Figure 9, the average transmission number slightly decreases with the increasing number of STAs, while the total transmission of the LTEWiFi coexistence system increases. Figure 10(a) shows that the average transmission number of CTCA increased by $21 \%$, $124 \%$, and $25 \%$ of those LTE-C, LAA, and LTE-U. Figure 10(b) shows CTCA can keep the average transmission number of LTE despite the increasing number of WiFi clients while the average transmission number equals that of LTE-C and is greater than LAA and LTE-U. Figure 11 shows that the average transmission number of CTCA is greater than the baselines when the duty cycle of LTE is larger than $20 \%$, while the average transmission number of CTCA is less than LTE-U and LTE-C when LTE duty cycle is less than $20 \%$. The main reason is that when LTE in a low duty cycle, there is nearly no parallel utilization of LTE and WiFi devices. 


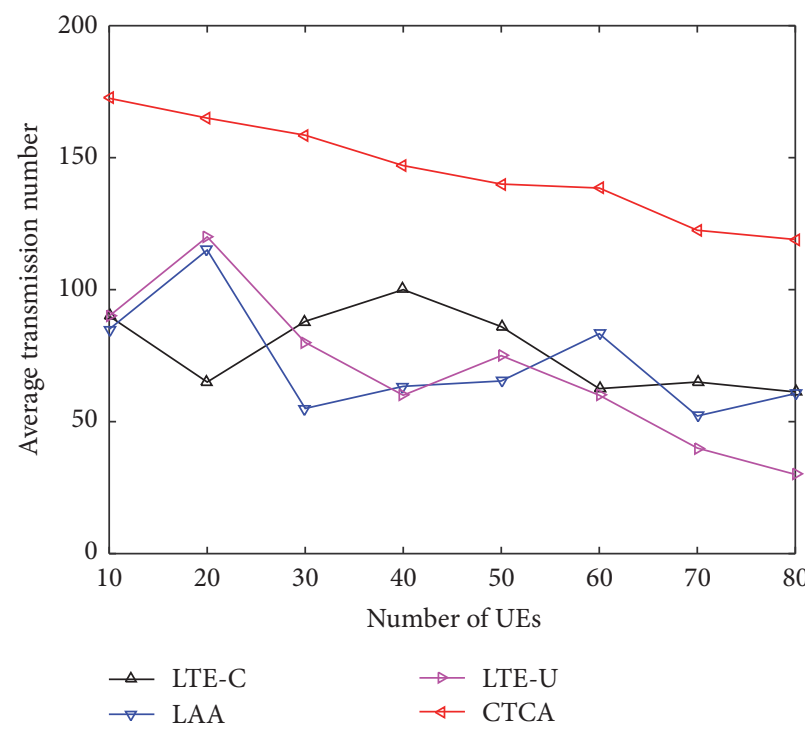

(a) Average transmission number and UE number
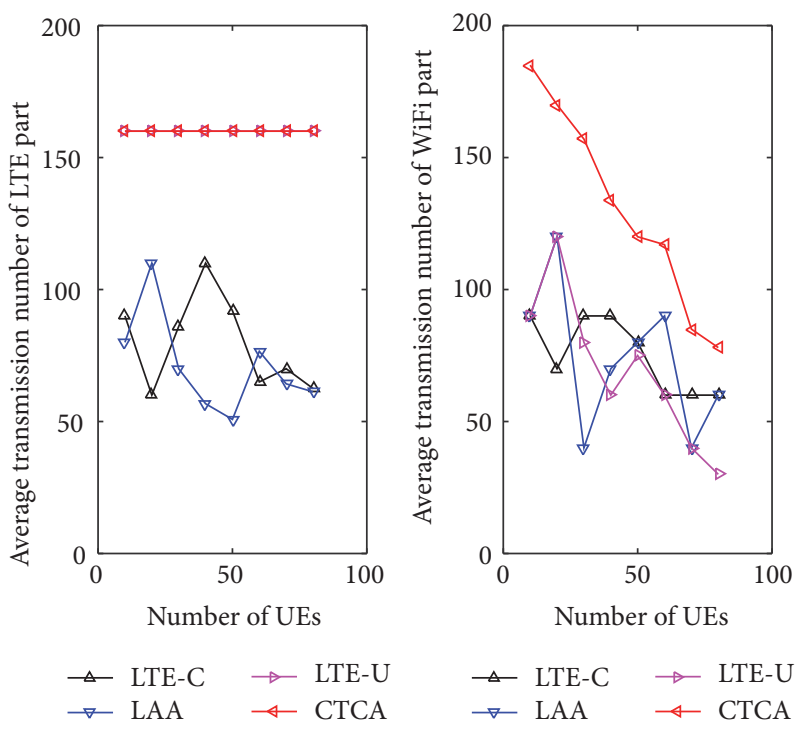

(b) Average transmission number of WiFi and LTE and UE number

FIGURE 9: Results of transmission number with different UE number.

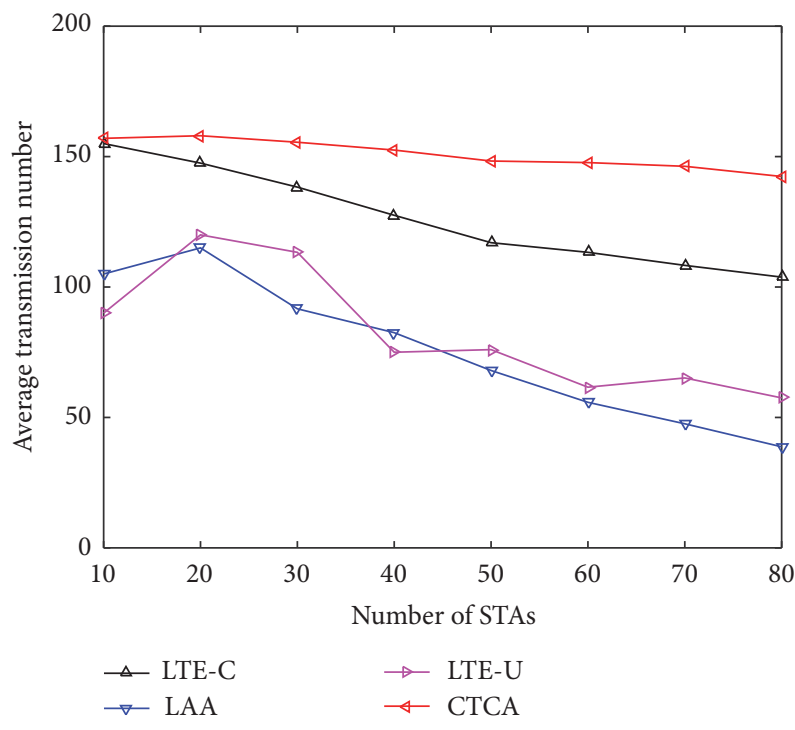

(a) Average transmission number and STA number
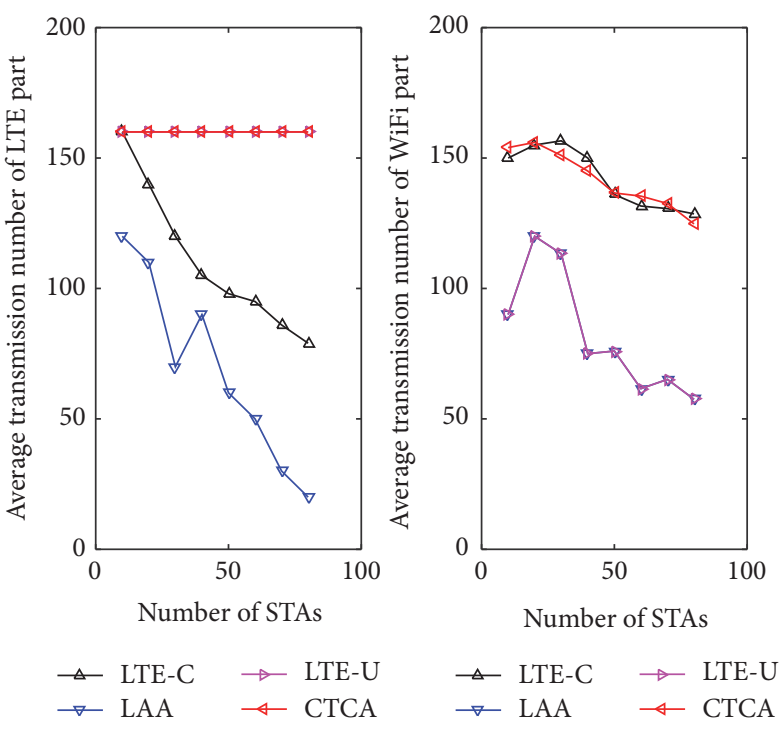

(b) Average transmission number of WiFi and LTE and STA number

FIGURE 10: Results of transmission number with STA number.

7.4.2. Average Transmission Delay. We also evaluate average transmission delay of CTCA, LTE-C, LAA, and LTE-U with different parameters. The results are shown in Figures 12, 13, and 14.

Figure 12 shows that the transmission delay of CTCA decreases by $10 \%, 39 \%$, and $29 \%$ compared with those of LTEC, LAA, and LTE-U. The reason why our algorithm could decrease the average transmission time delay is that relying on both LTE and WiFi devices could transmit the data if the traffic is demanded, while LTE-C, LAA, and LTE-U would cost some time units to get the right of the channel which causes additional time delay.

To further analyze the average transmission delay for WiFi and LTE part separately, we get the results which are showed in Figure 12(b). The average transmission delay of LTE devices increases with the number of UE clients since more UEs share the same resource, which can not guarantee that each of them could transmit in time. However, our approach is even better than LTE-C, LTE-U, and LAA.

We also evaluated the average transmission delay when the number of WiFi clients increases; the results are showed in Figure 13; the average transmission delay of CTCA decreases by $39 \%, 45 \%$, and $21 \%$ of those LTE-C, LAA, and LTE-U. Figure 13(b) also shows that the transmission time delay of LTE-C and LTE-U increases with the number of STAs.

Figure 14 shows that the transmission delay of CTCA is always lower than those of LTE-C, LAA, and LTE-U. This is because CTCA lets $\mathrm{WiFi}$ and LTE transmit together in 


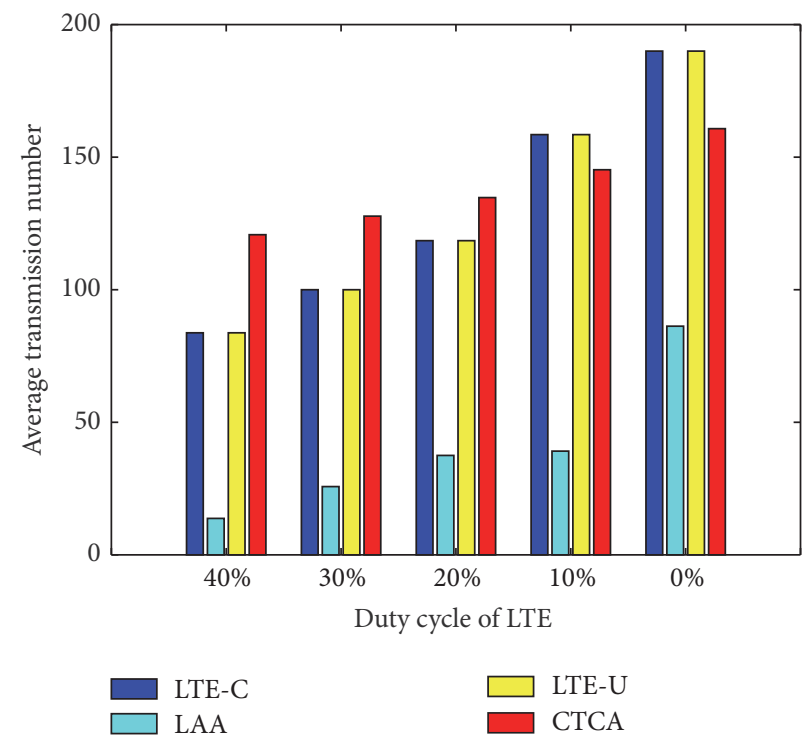

FIGURE 11: Results of transmission number with different duty cycle of LTE.

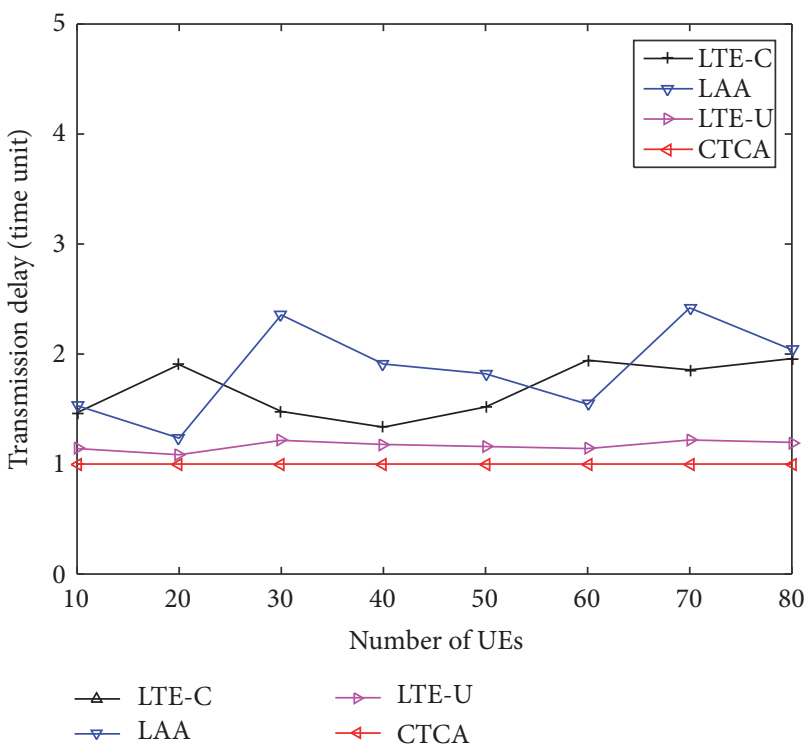

(a) Average transmission delay and UE number
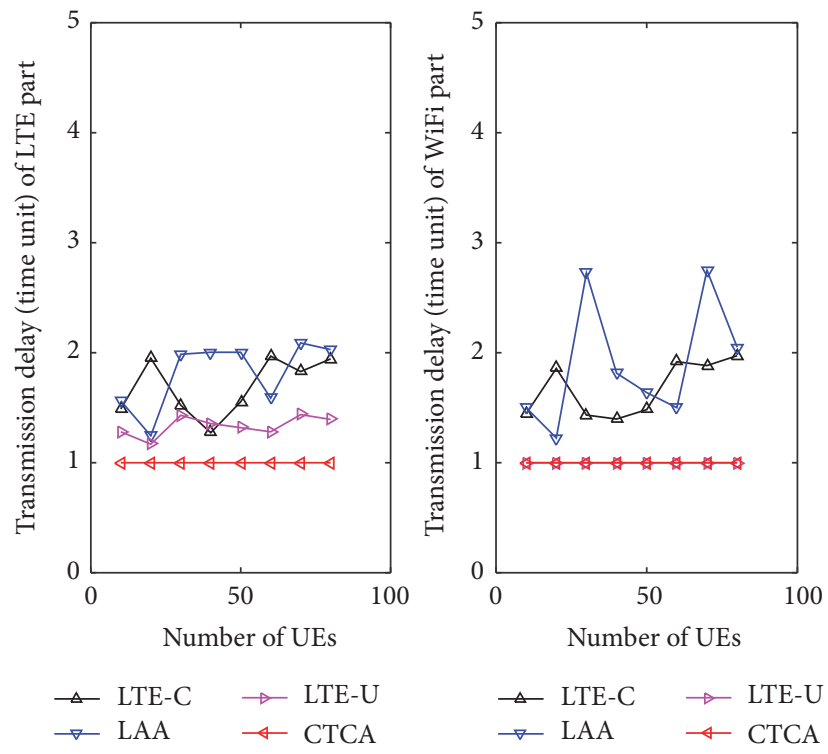

(b) Average transmission delay of WiFi and LTE and UE number

FIGURE 12: Results of transmission time delay with different UE number.

the same channel which could decrease the time delay for channel allocation. We also get another useful result that the average transmission delay could keep stable when the duty cycle of LTE is lower than $40 \%$.

7.4.3. Spectrum Efficiency. We evaluate the spectrum efficiency of CTCA in three scenarios; (i) UE is more denser than STA, (ii) STA is more denser than UE, and (iii) we also evaluate the average spectrum utilization when LTE duty cycle equals 40\%; Figure 15 shows that in scenario (i), the spectrum efficiency of CTCA is nearly $2 \mathrm{x}$ of LTE-C, LAA, and LTE-U; in scenario (ii), spectrum efficiency of CTCA is $1.2 \mathrm{x}, 2.3 \mathrm{x}$, and 2.2x of LTE-C, LAA, and LTE-U; in scenario (iii), spectrum efficiency is $1.3 \mathrm{x}, 3.4 \mathrm{x}$, and $2.9 \mathrm{x}$ of those LTEC, LAA, and LTE-U.

\section{Conclusion}

In this paper, we present Conflict-Tolerant Channel Allocation (CTCA) method in LTE and WiFi coexisting $5 \mathrm{GHz}$ band, especially for resolving the increase conflicts in dense deployment scenarios. To the best of our knowledge, this is the first Conflict-Tolerant Channel Allocation method for LTE and WiFi coexisting network aiming at efficient spectrum utilization and low time delay consideration. This work for the first time proposes Low Amplitude Stream Injection 


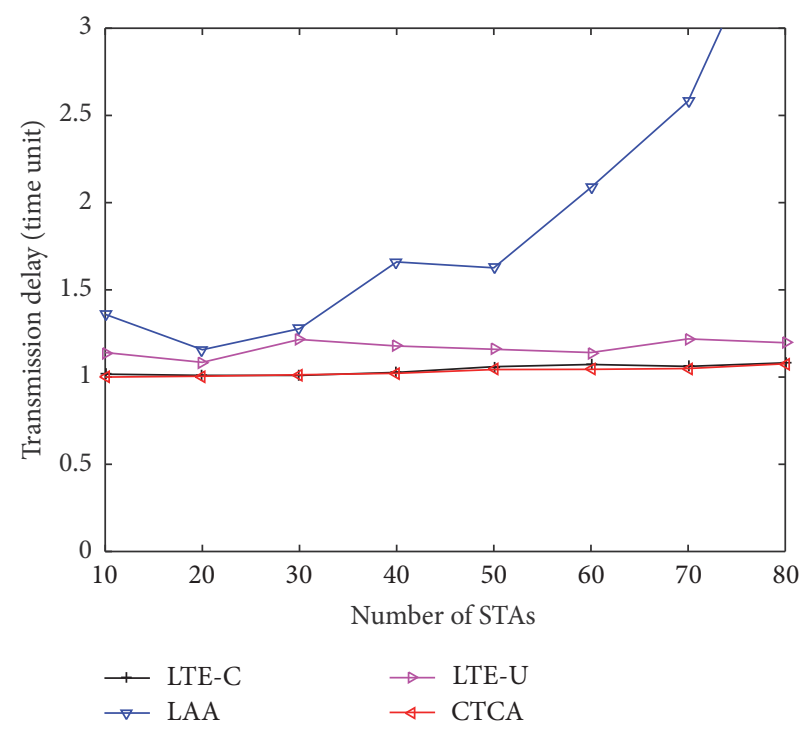

(a) Average transmission delay and STA number

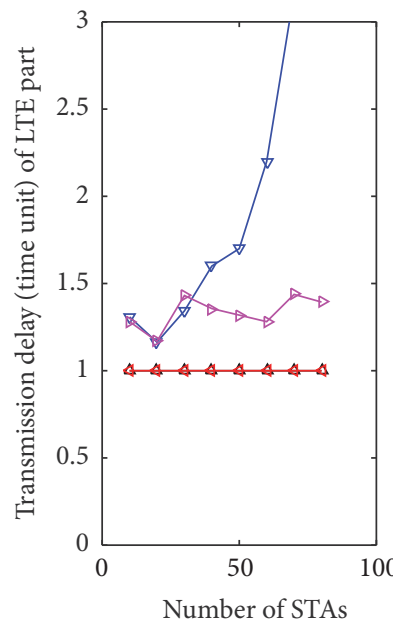

$\triangle$ LTE-C $\rightarrow$ LTE-U

$\rightarrow$ LAA $\neg$ CTCA

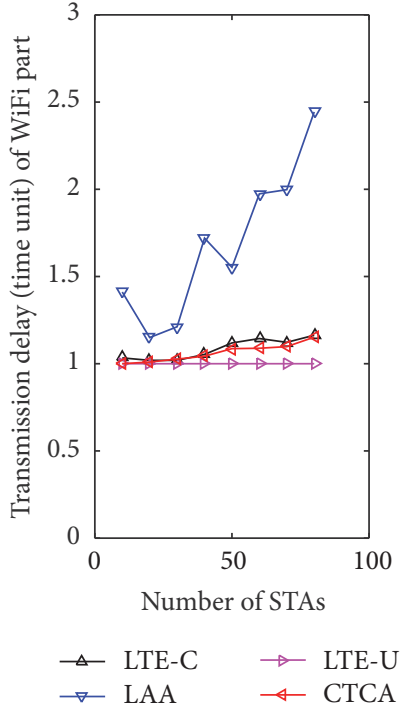

WiFi and LTE and STA number

FigURE 13: Results of transmission time delay with different STA number.

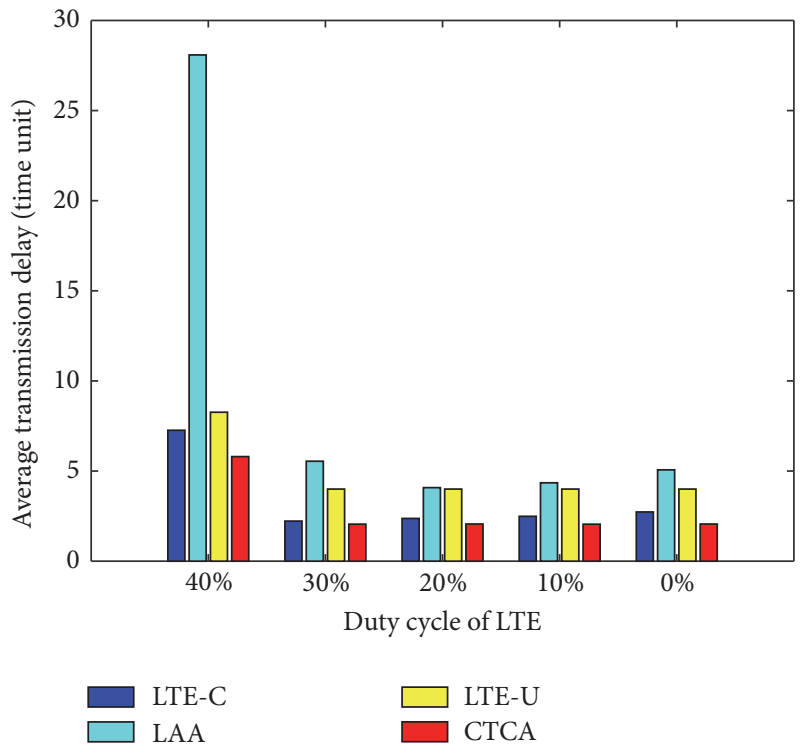

FIGURE 14: Results of transmission delay with different duty cycles of LTE.

(LASI) to deal with the increasing conflicts between LTE and WiFi especially in dense deployment scenarios. LASI method enables LTE and WiFi to transmit simultaneously by allocating the same channel with low transmission time delay and high spectrum efficiency. Cochannel allocation decision making of CTCA method defines a parameter to help AP decide whether to choose to have cochannel with another AP or other LTE base stations, by which the spectrum utilization efficiency could be further improved. In the future, we shall extend this work to achieve the fairness utilization of spectrum.

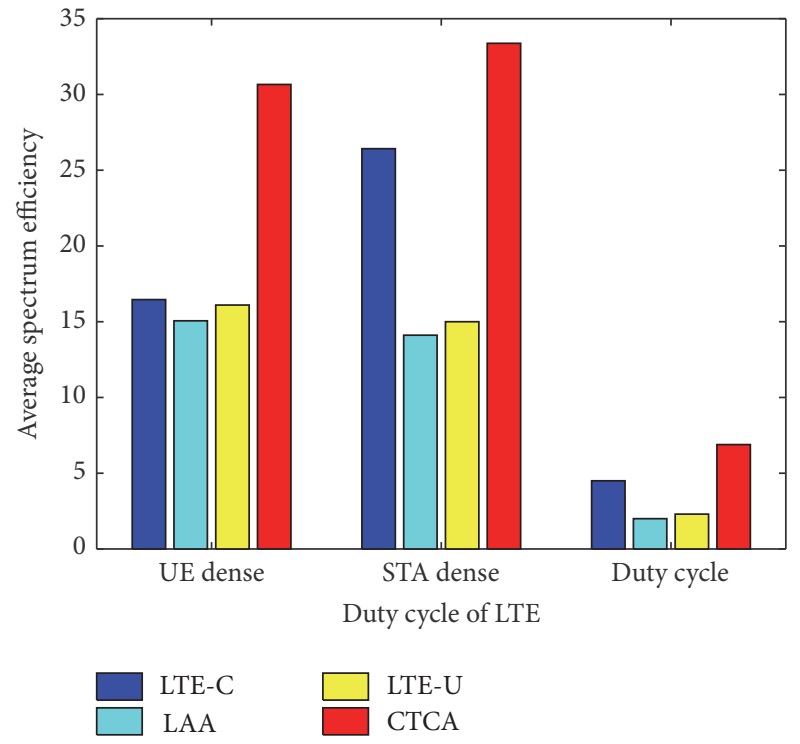

FIGURE 15: Results of spectrum utilization in different scenarios.

\section{Abbreviations}

$\mathrm{AP}_{i}: \quad$ Access point $i$ providing $\mathrm{WiFi}$ services in LTE/WiFi coexisting networks

$\mathrm{eNB}_{i}$ : eNodeB $i$ providing LTE services in LTE/WiFi coexisting networks

$\mathrm{STA}_{i k}$ : Client $k$ belonging to $\mathrm{AP}_{i}$

$\mathrm{UE}_{i k}$ : Client $k$ belonging to $\mathrm{eNB}_{i}$

$G_{w}: \quad$ Conflict graph between APs

$G_{l}: \quad$ Conflict graph between eNodeBs

$G_{w l}$ : Conflict graph between APs and eNodeB

G: $\quad G_{w} \cup G_{l} \cup G_{w l}$. 


\section{Competing Interests}

The authors declare that they have no competing interests.

\section{Acknowledgments}

This project is supported by NSF Grants CNS-1503590 and CNS-1539047.

\section{References}

[1] Cisco, "Cisco Visual Networking Index: Global Mobile Data Traffic Forecast Update, 2015-2020," 2016.

[2] "Spectrum monitoring," http://www.spectrummonitoring.com/ standards/\#LTE

[3] L. A. N. Man, S. Committee, and I. Computer, Wireless LAN Medium Access Control (MAC) and Physical Layer (PHY) Specifications, 2013.

[4] "Qualcomm wants LTE deployed on unlicensed spectrum," 2015.

[5] “U-LTE: Unlicensed Spectrum Utilization of LTE," 2015.

[6] "Ericsson first to give smartphone users indoor boost with License Assisted Access," 2015.

[7] Ericsson, T-Mobile push unlicensed LTE into limelight as ' $4.5 \mathrm{G}$ ' technology for IoT, 2015.

[8] “Overview of 3GPP Release 13 V0.0.6," 2014.

[9] M. Arunesh, B. Vladimir, and B. Suman, "A client-driven approach for channel management in wireless lans," in Proceedings of the 25th IEEE International Conference on Computer Communications (INFOCOM '06), Barcelona, Spain, April 2006.

[10] A. Mishra, S. Banerjee, and W. Arbaugh, "Weighted coloring based channel assignment for WLANs," ACM SIGMOBILE Mobile Computing and Communications Review, vol. 9, no. 3, pp. 19-31, 2005.

[11] K. Tan, J. Fang, Y. Zhang et al., "Fine-grained channel access in wireless lan," in Proceedings of the ACM SIGCOMM Conference (SIGCOMM '10), pp. 147-158, New Delhi, India, August 2010.

[12] T. Moscibroda, R. Chandra, Y. Wu, S. Sengupta, P. Bahl, and Y. Yuan, "Load-aware spectrum distribution in wireless lans," in Proceedings of the IEEE International Conference on Network Protocols (ICNP '08), IEEE, Orlando, Fla, USA, 2008.

[13] S. Rayanchu, V. Shrivastava, S. Banerjee, and R. Chandra, "FLUID: improving throughputs in enterprise wireless LANs through flexible channelization," in Proceedings of the 17th Annual International Conference on Mobile Computing and Networking (MobiCom'11) and Co-Located Workshops, pp. 1-12, Las Vegas, Nev, USA, September 2011.

[14] Qualcomm, "A comparison of LTE advanced HetNets and WIFI," 2014.

[15] S. Souvik, R. Božidar, L. Jeongkeun, and K. Kyu-Han, "Cspy: finding the best quality channel without probing," in Proceedings of the 19th Annual International Conference on Mobile Computing \& Networking (MobiCom '13), pp. 267-278, Miami, Fla, USA, October 2013.

[16] R. Chandra, R. Mahajan, T. Moscibroda, R. Raghavendra, and P. Bahl, "A case for adapting channel width in wireless networks," in Proceedings of the ACM SIGCOMM 2008 Conference on Data Communication (SIGCOMM '08), ACM, Seattle, Wash, USA, 2008.
[17] L. Yang, W. Hou, L. Cao, B. Y. Zhao, and H. Zheng, "Supporting demanding wireless applications with frequency-agile radios," in Proceedings of the 7th USENIX Conference on Networked Systems Design and Implementation (NSDI '10), p. 5, San Jose, Calif, USA, April 2010.

[18] H. Yu, O. Bejarano, and L. Zhong, "Combating Inter-cell Interference in 802.1lac-based multi-user MIMO networks," in Proceedings of the 20th ACM Annual International Conference on Mobile Computing and Networking (MobiCom '14), pp. 141152, Maui, Hawaii, USA, September 2014.

[19] L. Yang, W. Hou, L. Cao, B. Y. Zhao, and H. Zheng, "Supporting Demanding Wireless Applications with Frequency-agile Radios Multimedia Streaming in Home/Office," pp. 1-21, 2010.

[20] E. Ziouva and T. Antonakopoulos, "Reprint CSMA/CA performance under high traffic conditions: throughput and delay analysis throughput and delay analysis," Computer Communications, vol. 25, no. 3, pp. 313-321, 2002.

[21] J. Herzen, A. Banchs, V. Shneer, and P. Thiran, "CSMA/CA in time and frequency domains," in Proceedings of the International Conference on Network Protocols (ICNP '15), San Francisco, Calif, USA, 2015.

[22] S. Gollakota, F. Adib, D. Katabi, and S. Seshan, "Clearing the RF smog: making $802.11 n$ robust to cross-technology interference," in Proceedings of the ACM SIGCOMM Conference, Toronto, Canada, August 2011.

[23] Y. Yan, P. Yang, X.-Y. Li, Y. Tao, L. Zhang, and L. You, “ZIMO: building cross-technology MIMO to harmonize ZigBee Smog with WiFi flash without intervention categories and subject descriptors experiment rationale and setup," in Proceedings of the 19th Annual International Conference on Mobile Computing and Networking (MobiCom '13), Miami, Fla, USA, 2013.

[24] "Lte in unlicensed spectrum: Harmonious coexistence with wifi," 2015.

[25] A. M. Cavalcante, E. Almeida, R. D. Vieira et al., "Performance evaluation of LTE and Wi-Fi coexistence in unlicensed bands," in Proceedings of the IEEE 77th Vehicular Technology Conference (VTC Spring '13), Dresden, Germany, June 2013.

[26] B. Jia and M. Tao, "A channel sensing based design for LTE in unlicensed bands," in Proceedings of the IEEE International Conference on Communication Workshop (ICCW'15), June 2015.

[27] C. Cano and D. J. Leith, "Coexistence of WiFi and LTE in unlicensed bands: a proportional fair allocation scheme," in Proceedings of the IEEE International Conference on Communication Workshop (ICCW '15), pp. 2288-2293, IEEE, London, UK, June 2015.

[28] O. R. Fitness, F. O. R. Any, P. Purpose, A. L. L. Such, and I. Is, “Coexistence Study for LTE-U SDL V1.0 (2015 -02)," Tech. Rep., 2015.

[29] F. M. Abinader, E. P. L. Almeida, F. S. Chaves et al., "Enabling the coexistence of LTE and Wi-Fi in unlicensed bands," IEEE Communications Magazine, vol. 52, no. 11, pp. 54-61, 2014.

[30] M. G. Sriyananda, I. Parvez, I. Güvene, M. Bennis, and A. I. Sarwat, "Multi-armed bandit for LTE-U and WiFi coexistence in unlicensed bands," in Proceedings of the IEEE Wireless Communications and Networking Conference (WCNC '16), Doha, Qatar, April 2016.

[31] Z. Jiang and S. Mao, "Inter-operator opportunistic spectrum sharing in LTE-unlicensed," IEEE Transactions on Vehicular Technology, no. 99, 2016.

[32] C. Chen, R. Ratasuk, and A. Ghosh, "Downlink performance analysis of LTE and WiFi coexistence in unlicensed bands with 
a simple listen-before-talk scheme," in Proceedings of the 81st IEEE Vehicular Technology Conference (VTC Spring '15), pp. 1-5, IEEE, May 2015.

[33] A. Bhorkar, C. Ibars, and P. Zong, "On the throughput analysis of LTE and WiFi in unlicensed band," in Proceedings of the 48th Asilomar Conference on Signals, Systems and Computers (ACSSC '15), pp. 1309-1313, Pacific Grove, Calif, USA, November 2014.

[34] J. Jeon, H. Niu, Q. Li, A. Papathanassiou, and G. Wu, "LTE with listen-before-talk in unlicensed spectrum," in Proceedings of the IEEE International Conference on Communication Workshop (ICCW'15), pp. 2320-2324, June 2015.

[35] P. Xia, Z. Teng, and J. Wu, "How loud to talk and how hard to listen-before-talk in unlicensed LTE," in Proceedings of the IEEE International Conference on Communication Workshop (ICCW '15), pp. 2314-2319, London, UK, June 2015.

[36] H. Li, Y. Chang, F. Hao, A. Men, J. Zhang, and W. Quan, "Study on dynamic channel switch in license-assisted-access based on listen-before-talk," in Proceedings of the 13th International Symposium on Wireless Communication Systems (ISWCS '16), pp. 506-510, Poznań, Poland, September 2016.

[37] Q. Chen, G. Yu, R. Yin, A. Maaref, G. Y. Li, and A. Huang, "Energy efficiency optimization in licensed-assisted access," IEEE Journal on Selected Areas in Communications, vol. 34, no. 4, pp. 723-734, 2016.

[38] T. Yang, C. Guo, S. Zhao, Q. Zhang, and Z. Feng, "Channel occupancy cognition based adaptive channel access and backoff scheme for LTE system on unlicensed band," in Proceedings of the IEEE Wireless Communications and Networking Conference (WCNC '16), pp. 1-6, IEEE, Doha, Qatar, April 2016.

[39] S. Han, Y. C. Liang, Q. Chen, and B. H. Soong, "Licensedassisted access for lte in unlicensed spectrum: a mac protocol design," IEEE Journal on Selected Areas in Communications, vol. 34, no. 10, pp. 2550-2561, 2016.

[40] E. Chai, K. Sundaresan, M. A. Khojastepour, and S. Rangarajan, "LTE in unlicensed spectrum: are we there yet?" in Proceedings of the 22nd Annual International Conference on Mobile Computing and Networking (MobiCom '16), pp. 135-148, ACM, New York, NY, USA, 2016.

[41] L. Li, A. H. Jafari, X. Chu, and J. Zhang, "Simultaneous transmission opportunities for LTE-LAA smallcells coexisting with WiFi in unlicensed spectrum," in Proceedings of the IEEE International Conference on Communications (ICC '16), pp. 1-7, May 2016.

[42] S. Yun and L. Qiu, "Supporting WiFi and LTE co-existence," in Proceedings of the IEEE Conference on Computer Communications (INFOCOM '15), pp. 810-818, IEEE, Hong Kong, May 2015. 


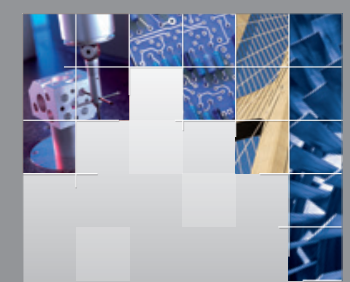

\section{Enfincering}
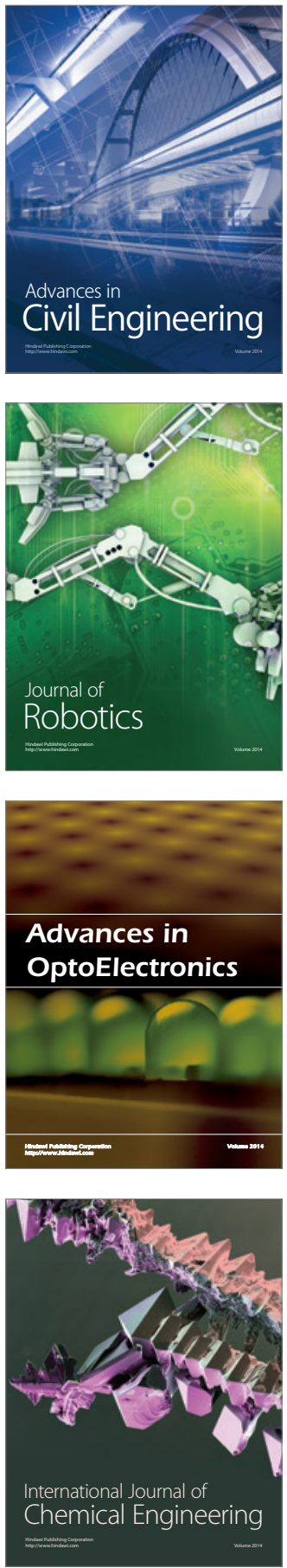

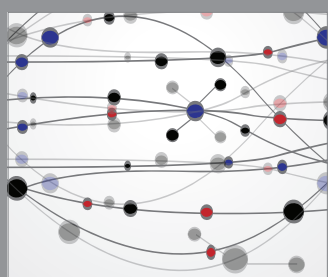

The Scientific World Journal

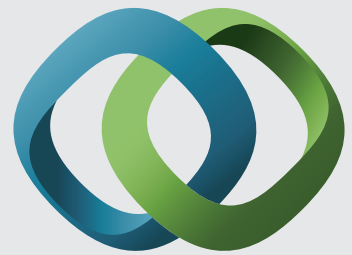

\section{Hindawi}

Submit your manuscripts at

https://www.hindawi.com
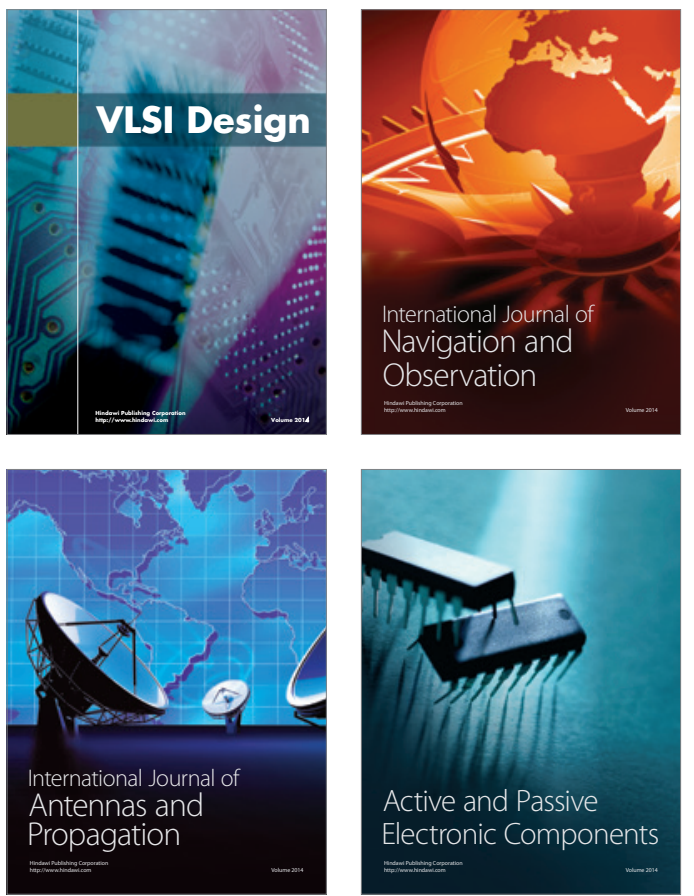
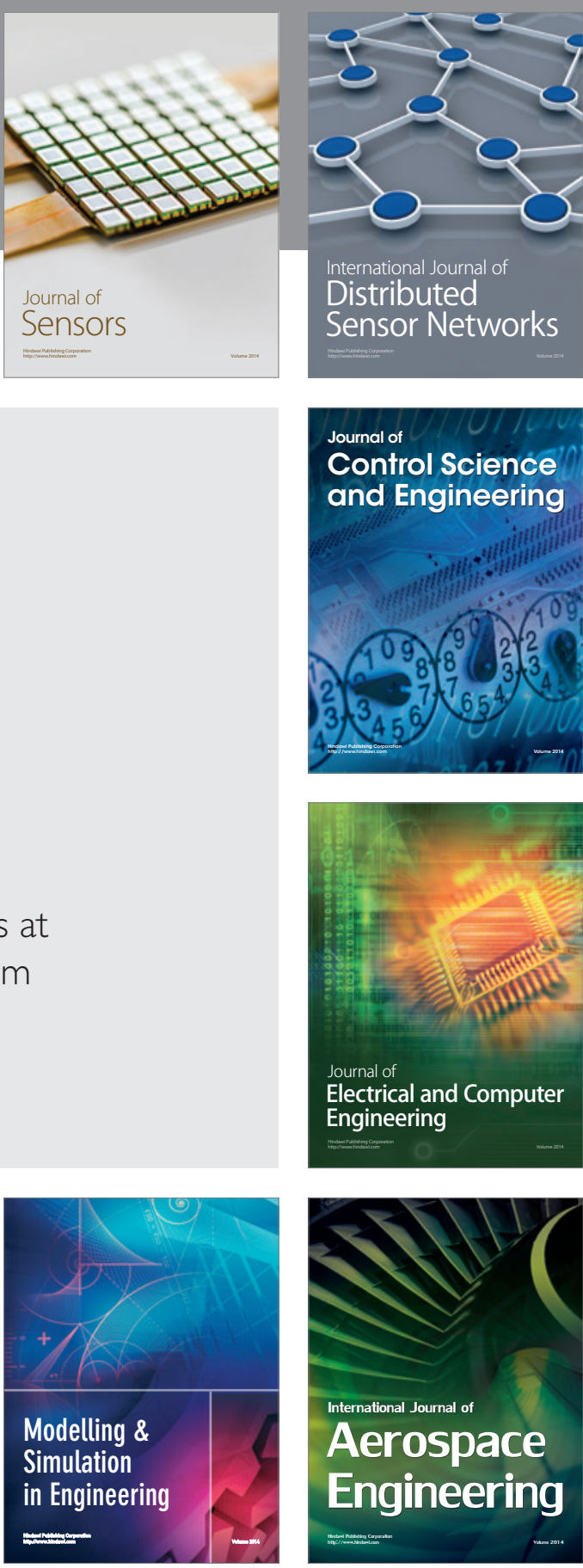

International Journal of

Distributed

Sensor Networks

$-$

Joumal of

Control Science

and Engineering
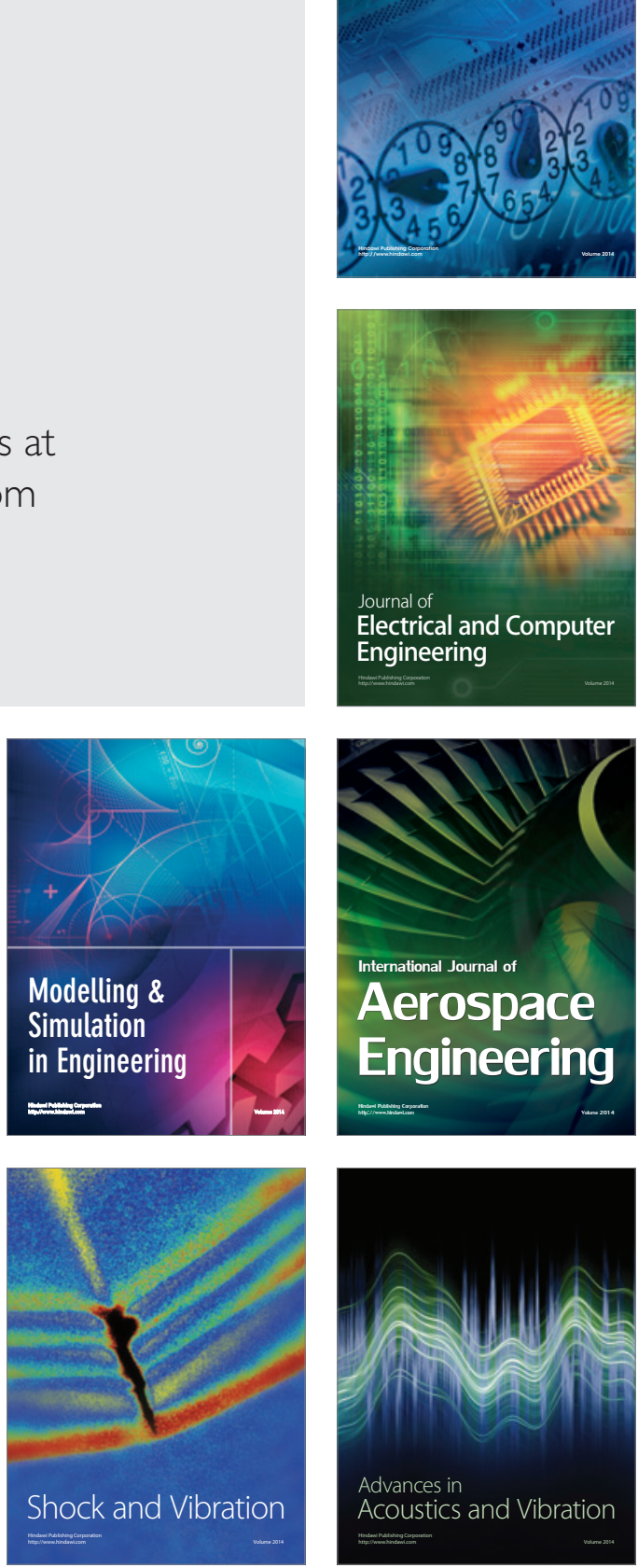